\title{
Upscaling mesoscopic simulation results to develop constitutive relations for macroscopic modeling of equiaxed dendritic solidification
}

\author{
M. Torabi Rad ${ }^{1}$, M. Založnik ${ }^{2}$, H. Combeau ${ }^{2}$, and C. Beckermann ${ }^{1}$ \\ ${ }^{1}$ Department of Mechanical Engineering, University of Iowa, Iowa City, IA 52242, USA \\ ${ }^{2}$ Université de Lorraine, CNRS, IJL, F-54000 Nancy, France
}

Corresponding Author: M. Torabi Rad' (mahdi-torabirad@uiowa.edu)

Keywords: binary alloys, dendritic solidification, grain growth, macro and meso scales, constitutive equations

\begin{abstract}
Macroscale solidification models incorporate the microscale and mesoscale phenomena of dendritic grain growth using constitutive relations. These relations can be obtained by simulating those phenomena inside a Representative Elementary Volume (REV) and then upscaling the results to the macroscale. In the present study, a previously developed mesoscopic envelope model is used to perform three-dimensional simulations of equiaxed dendritic growth at a spatial scale that corresponds to a REV. The mesoscopic results are upscaled by averaging them over the mesoscopic simulation domain. The upscaled results are used to develop new constitutive relations, which, unlike the currently available relations, do not rely on highly simplified assumptions about the grain envelope shape or the solute diffusion conditions around it. The relations are verified by comparing the predictions of the macroscopic model with the upscaled
\end{abstract}

\footnotetext{
1 Present address: Access e.V., Intzestraße 5, D-52072, Aachen, Germany, Tel: +49 02418098016 , Email: m.torabirad@access.rwth-aachen.de
} 
mesoscopic results at different solidification conditions. These relations can now be used in macroscopic models of equiaxed solidification to incorporate more realistically the microscale and mesoscale phenomena.

\section{Introduction}

Solidification is a complex multiscale problem that is controlled by phenomena occurring at length scales that are distinct from each other and range over roughly five orders of magnitude $[1,2]$. At the macroscale (i.e., the scale of the whole casting) heat transfer and typically melt convection take place, grains can move, and the solid might deform. At the mesoscale (i.e., the scale of the primary dendrite arms spacing ranging from 1 to $0.1 \mathrm{~mm}$ ) grains grow controlled by solute and heat diffusion and under the influence of collective interactions; this determines the final grain structure. At the microscale (i.e., the scale of a dendrite tip radius ranging from $10^{-2}$ to $10^{-3} \mathrm{~mm}$ ) the competition between the microscale heat/solute diffusion and surface tension determines the dendrite tip radius and velocity. What makes solidification modeling a complex task is that there is a strong inter-scale coupling between the phenomena occurring at the different length scales. For example, macroscale melt convection influences the microscale solute diffusion, and is, itself, influenced by the microscopic structure of the semi-solid mush. Because of this coupling, a model that simulates the macroscale behavior of a solidifying system needs to incorporate the microscale and mesoscale phenomena. Incorporating these phenomena by directly simulating them will, however, require having computational cells as small as one micrometer in the simulation domain that can be as large as few meters. This will result in having millions of cells in each direction. The computational cost of such a simulation will continue to remain beyond the reach of computer powers in the foreseeable future. Therefore, one needs to find another way to incorporate the microscale and mesoscale phenomena in models that simulate the macroscale behavior. 
Microscale and mesoscale phenomena can be incorporated in the models that simulate the macroscale behavior using volume-averaging methods. Averaging concepts were first applied in the solidification field by Beckermann and Viskanta [3] in the mid to late 1980s and later significantly extended by $\mathrm{Ni}$ and Beckermann [4] and Wang and Beckermann [5-9]. Volumeaveraging is now a widely accepted method in developing macroscale solidification models as is indicated by more than one thousand citations to the original papers. Volume-averaged macroscale models have been used to simulate solidification in systems as large as steel ingots [10-12]. It is beyond the scope of this paper to review the governing equations in detail, but thorough reviews are available $[6,13]$. In brief, these models are derived by averaging the local equations (i.e., equations that are valid at the microscopic scale) for each phase over a volume that contains all the phases present in the system and is called the Representative Elementary Volume (REV). The size of an REV must be small compared to the size of the entire system, but large compared to the scale on which the microscale phenomena takes place. The resulting volume-averaged equations contain phase fractions and source terms. These source terms, which account for the microscale and mesoscale transport phenomena occurring at the interfaces between the different phases, depend on variables that are not predicted by the macroscopic model, because the lower scale information that these variables represent has been lost in the averaging process. Accurate calculation of these source terms, therefore, requires one to do a formal analysis on the REV scale and then pass up the information to the macroscale, through constitutive relations, in a process called upscaling. The term upscaling simply means that in the ladder of length scales information is passed up from a smaller scale to a larger scale by averaging. This upscaling has never been tried in the field of solidification, mainly because of the complexity that arises as the result of the large range of length scales that need to be resolved. In other words, in solidification, there is a large gap between the involved micro and macro length scales. Therefore, the currently available constitutive relations have been based on somewhat simplistic assumptions rather than a formal analysis of the REV scale. 
The gap between the micro and macro scales can be bridged using the mesoscopic model originally developed for pure materials by Steinbach et al. [1,2], extended for binary alloys by Delaleau et al. [14], and further validated by Souhar et al. [15] by performing three-dimensional simulations of equiaxed growth and comparing the results with experimental scaling laws [16]. Mesoscopic models directly resolve the transport phenomena on the REV scale, by solving an equation for the heat/solute transport on this scale, and incorporate microscale phenomena, by using a local analytical solution for the microscale heat/solute transport. The computational power requirement of these models is significantly lower than the models that resolve the microscale phenomena directly, such as the phase field models $[1,2]$. This allows one to do three-dimensional simulations at low undercoolings, corresponding to realistic process conditions, and at relatively large domain sizes that correspond to a REV.

In this paper, the mesoscopic envelope model of Delaleau et al. [14] is used to perform threedimensional simulations of equiaxed growth on a spatial scale that corresponds to a REV. Simulations are performed for several initial undercoolings and grain densities and the results were upscaled by averaging them over the volume of the REV. The upscaled results are examined in detail and used to develop new, more accurate constitutive relations for macroscale solidification models. The new constitutive relations are verified by comparing the predictions of the volumeaveraged macroscopic model with the upscaled mesoscopic results at different solidification conditions.

The paper is organized as follows: The macroscopic model is introduced in section 2. A brief introduction of the mesoscopic model and mesoscopic results are presented in section 3 . The constitutive relations are developed in section 4 and are verified in section 5. 


\section{Volume-averaged Macroscopic Model}

In this section, the conservation equations of the volume-averaged macroscopic model used in the present study are first introduced. It is shown that these equations contain variables that need to be obtained from constitutive relations. The constitutive relations are discussed next.

\subsection{Conservation Equations}

Following the pioneering work of Wang and Beckermann [5-9], to develop a macroscopic model for equiaxed solidification in an undercooled melt, a solidifying system is first assumed to consist of three phases: solid, inter-dendritic liquid, and extra-dendritic liquid. The two liquid phases are separated by the grain envelope, which is a virtual and smooth surface that connects the primary tips and the tips of actively growing secondary arms. A secondary arm is defined as active when it is longer than the next active secondary arm closer to the primary tip. Two liquid phases are introduced in the model because the solute diffusion is governed by length scales of different orders of magnitude: the secondary arm spacing in the inter-dendritic liquid and the distance between grains in the extra-dendritic liquid. Figure 1 shows a schematic of a grain envelope and the regions of the solid, inter-dendritic liquid, and extra-dendritic liquid phases, denoted by $s, d$, and $e$, respectively. Writing the local (i.e., the microscopic level) equation for the mass conservation in the extra-dendritic liquid in the absence of melt convection and solid motion, and using the averaging theorems discussed in detail in Wang and Beckermann [5] to average that equation over the volume of the REV, $V_{0}$, results in the following volume-averaged equation for the average growth kinetics:

$$
\frac{d g_{e n v}}{d t}=\frac{1}{V_{0}} \iint_{A_{e n v}} \boldsymbol{w}_{e n v} \cdot \boldsymbol{n} d A=S_{e n v} w_{e n v}
$$


where $g_{e n v}, A_{e n v}, w_{e n v}, n, S_{e n v}$, and $w_{e n v}$ are the envelope volume fraction (i.e., grain fraction), envelope surface area, local envelope growth velocity vector, unit vector normal to the envelope surface and pointing outside the envelope, envelope surface area per unit volume of the REV (i.e., $\left.A_{e n v} / V_{0}\right)$, and average envelope growth velocity, respectively. This equation indicates that the envelope volume fraction $g_{e n v}$ will increase, in other words growth will continue, as long as $w_{e n v}$ is greater than zero. The terms on the left-hand and right-hand sides of the first equality represent the change in the mass inside the envelope and the net rate of mass exchange at the envelope surface.

Writing the local equation for the solute conservation in the extra-dendritic liquid and following a procedure similar to the one discussed above equation (1) gives the volume-averaged equation for the average solute diffusion rates from the dendrite envelopes as:

$$
\frac{\partial}{\partial t}\left(g_{e} \bar{C}_{e}\right)=-\frac{1}{V_{R E V}} \iint_{A_{e n v}} C_{l}^{*} \boldsymbol{w}_{e n v} \cdot \boldsymbol{n} d A+\frac{1}{V_{R E V}} \iint_{A_{e n v}} \boldsymbol{j}_{e} \cdot \boldsymbol{n} d A=\frac{\partial g_{e}}{\partial t} C_{l}^{*}+S_{e n v} \frac{D_{l}}{\delta_{e n v}}\left(C_{l}^{*}-\bar{C}_{e}\right)
$$

where $g_{e}=1-g_{e n v}, \bar{C}_{e}, C_{l}^{*}, \boldsymbol{j}_{e}, D_{l}$ and $\delta_{e n v}$ are the extra-dendritic liquid fraction, average solute concentration in the extra-dendritic liquid, equilibrium solute concentration in the liquid, solute diffusion flux in the extra-dendritic liquid, solute mass diffusivity in the liquid, and average diffusion length around the envelopes, respectively. Note that, on the right-hand side of the first equality, the negative and positive signs of the first and second terms, respectively, reflect the fact that the unit vector $n$ is defined to be pointing outside the envelope. These terms represent the microscopic solute transfer (from the inter-dendritic to extra-dendritic) at the envelope surface. The first term represents the solute transfer due to the growth of the envelope and can be simply substituted using the first equality in equation (1) (note that $C_{l}^{*}$ is assumed to be uniform in the 
REV and be, therefore, taken outside the integral). The second term represents the solute transfer due to the solute diffusion; the integral in this term can be modeled as the product of the envelope specific surface area $S_{e n v}$ and a mean diffusive flux at the envelope surface. This flux can be assumed to be directly proportional to the driving force for diffusion, which is the difference between the solute concentration in the extra-dendritic liquid adjacent to the envelope and the average solute concentration in the extra-dendritic liquid (i.e., $C_{l}^{*}-\bar{C}_{e}$ ), and inversely proportional to the average diffusion length around the envelopes $\delta_{e n v} ; \delta_{e n v}$ is a measure of how far the solute has diffused away from the envelope. To better understand the concept of diffusion length, one can look at Figure 1, where a schematic of the solute distribution in the extra-dendritic liquid ahead of the primary tips is shown. The green line in the plot shows the tangent to the profile at the primary tip. The tangent intersects with the horizontal dashed line representing $\bar{C}_{e}$, at a distance that is proportional to the envelope diffusion length $\delta_{e n v}$. Finally, the term $C_{l}^{*}-\bar{C}_{e}$ is linked to the average undercooling in the extra-dendritic liquid, which is the driving force for growth.

In equations (1) and (2), the variables $S_{e n v}, w_{e n v}$, and $\delta_{e n v}$ need to be obtained from constitutive relations. The next section discusses the procedure to derive these relations and also the assumptions that have been commonly used in the literature to derive the currently available constitutive relations.

\subsection{Constitutive Relations}

To obtain the constitutive relations for the envelope variables $S_{e n v}, w_{e n v}$, and $\delta_{e n v}$, the envelope is first approximated by the volume-equivalent sphere, referred to as sphere hereafter. A schematic of the sphere is also shown in Figure 1. Then, the envelope variables are related to the sphere variables as follows. 


\subsubsection{Relating envelope variables to sphere variables}

The envelope surface area per unit volume of the REV, $S_{e n v}$, is related to the sphere surface area per unit volume of the REV, $S_{s p}$, directly from the definition of the envelope sphericity $\psi$

$$
S_{e n v}=\frac{S_{s p}}{\psi}
$$

One should note that the sphericity $\psi$ is a purely geometrical variable (i.e., it depends solely on the geometry of the envelope). The sphericity of a sphere is equal to unity by definition, and any other shape has a sphericity less than unity (for example, the sphericity of an octahedron is 0.85 [17]).

To relate the average envelope velocity, $w_{e n v}$ to the sphere growth velocity, $w_{s p}$, one needs to recognize that equation (1) holds for any shape; since the volume of an envelope and its sphere are equal, the time derivative of envelope volume fraction and sphere volume fraction will be equal and one can, therefore, write $S_{e n v} w_{e n v}=S_{s p} w_{s p}$. In this relation, $S_{e n v}$ can be substituted from equation (3) to give:

$$
w_{e n v}=\psi w_{s p}
$$

Next, the variation of $\psi$ during growth is discussed. Equiaxed growth starts from a spherical nucleus, which has $\psi=1$ and, from equation (4), $w_{e n v} / w_{s p}=1$. As the spherical nucleus grows into the undercooled melt surrounding it, its shape becomes unstable and relatively fast growth along the energetically favorable crystallographic directions, compared to growth along the other directions, gradually transitions the shape into a dendrite, which has $\psi<1$ and, again from 
equation (4), $w_{e n v} / w_{s p}<1$. Therefore, during growth, $\psi$ and $w_{e n v} / w_{s p}$ decrease from their initial value of unity.

In the current literature, there are no relations to predict the decrease in $\psi$ or $w_{e n v} / w_{s p}$ during growth. Therefore, macroscopic models had to rely on pre-determined and constant values for $\psi$ and $w_{e n v} / w_{s p}$. For example, in the study of Martorano et al. [18], $\psi$ and $w_{e n v} / w_{s p}$ have been assumed to be equal to unity during the entire growth period; in other words, it is assumed that grains retain their initial spherical shape. In the studies of Appolaire et al. [19] and of Ludwig and $\mathrm{Wu}[20,21], \psi$ is assumed to be equal to 0.85 (i.e., the sphericity of an octahedron) and $w_{e n v} / w_{s p}$ is assumed to be equal to the sphericity. Disregarding the decrease in $\psi$ and $w_{e n v} / w_{s p}$ during growth can be expected to result in inaccuracies in the macroscopic models. In fact, Rappaz and Thevoz [22], compared the cooling curves measured in the experiments with the ones predicted by their solute diffusion model and noticed that their model does not predict the recalescence very well. They attributed this partly to the fact that in their model, sphericity was assumed to be equal to unity during the entire growth. As another example, Wu et al. [23, 24] did columnar to equiaxed transition (CET) simulations with different values for sphericity and found that the CET position is highly sensitive to the sphericity value. Developing a relation to predict the decreases in $\psi$ and, therefore, in $w_{e n v} / w_{s p}$, during growth is one of the objectives of this study.

To relate $\delta_{e n v}$ to the sphere diffusion length, $\delta_{s p}$, one needs to realize that the envelope diffusion length is determined by the diffusion field around the envelope. It is therefore, in general, a complicated function of the envelope shape, size and growth velocity and a relation between $\delta_{e n v}$ and $\delta_{s p}$ cannot be obtained from a simple and purely geometrical analysis, such as the one we did to obtain equation (4). Such a relation has never been proposed in the literature mainly because the complex nature of solute diffusion field around an envelope precludes one from finding an analytical relation for $\delta_{e n v}$. Macroscopic models, therefore, have simply assumed $\delta_{e n v}=\delta_{s p}[5-9$, 
18, 25]. This assumption might have reasonable accuracy during the initial stages of growth, when the envelope is spherical; however, as the envelope becomes dendritic with growth, the assumption can be expected to become increasingly inaccurate. Developing a relation for $\delta_{e n v}$ is another objective of this study.

\subsubsection{Relations for Sphere Variables}

In the previous section, the envelope variables were related to the sphere variables. In this section, the relations for the sphere variables are outlined first and then interesting limiting cases of the relation for $\delta_{s p}$ are discussed.

The sphere surface area per unit volume of the REV, $S_{s p}$, is calculated from

$$
S_{s p}=\frac{4 \pi n R_{s p}^{2}}{V_{0}}=\frac{4 \pi n R_{s p}^{2}}{\left(4 \pi n R_{s p}^{3} / 3\right) / g_{e n v}}=\frac{3 g_{e n v}}{R_{s p}}
$$

where $n$ and $R_{s p}$ are the effective number of grains and the sphere radius, respectively. Note that, in this equation, the first equality simply follows from the definition of $S_{s p}$ and the second equality follows from the definition of $g_{e n v}$ (i.e., the ratio of the total envelope volume to the REV volume $V_{0}$ ) and the fact that the envelope volume is equal to the sphere volume. The sphere radius $R_{s p}$ is calculated from

$$
\frac{d R_{s p}}{d t}=w_{s p}
$$

Next, the model needs a relation for $w_{s p}$. Currently, macroscopic models assume simple fixed envelope geometries and therefore obtain $w_{\text {sp }}$ from the primary tip velocity, $V_{\mathrm{t}}$, multiplied by a 
constant geometrical factor [7, 17-24]. In reality, $w_{\mathrm{sp}}$ depends on the velocity of the primary and secondary tips, and on the envelope shape. Developing a relation for $w_{s p}$ that accounts for the realistic evolving envelope shape is one of the objectives of the present study.

The sphere diffusion length $\delta_{s p}$ is calculated from the relation developed by Martorano et al. [18]

$$
\begin{aligned}
\frac{\delta_{s p}}{R_{s p}}=\frac{R_{s p}}{R_{f}^{3}-R_{s p}^{3}}\left\{\left(\frac{R_{f} R_{s p}}{P e_{s p}}+\frac{R_{s p}^{2}}{P e_{s p}^{2}}-R_{f}^{2}\right) e^{-P e_{s p}\left(\frac{R_{f}}{R_{s p}}-1\right)}-\left(\frac{R_{s p}^{2}}{P e_{s p}}+\frac{R_{s p}^{2}}{P e_{s p}^{2}}-\frac{R_{f}^{3}}{R_{s p}}\right)\right. \\
\left.+P e_{s p} \frac{R_{f}^{3}}{R_{s p}}\left\{e^{-P e_{s p}\left(\frac{R_{f}}{R_{s p}}-1\right)} \frac{\operatorname{Iv}\left(P e_{s p} R_{f} / R_{s p}\right)}{P e_{s p} R_{f} / R_{s p}}-\frac{\operatorname{Iv}\left(P e_{s p}\right)}{P e_{s p}}\right)\right\}
\end{aligned}
$$

where $P e_{s p}=w_{s p} R_{s p} / D_{l}$ is the sphere growth Péclet number, $R_{f}$ is the final grain radius, and Iv ( ) is the Ivantsov function. This equation indicates that the diffusion length around a sphere depends on the radius and growth velocity of the sphere and the final grain radius. A better insight into this dependence can be obtained by simplifying equation (7) in two interesting limiting cases: the high $P e_{s p}$ limit and the high $R_{f}$ limit (i.e., the single grain limit). This is discussed next.

In the high $P e_{s p}$ limit, $e^{-P e_{s p}\left[\left(R_{f} / R_{s p}\right)-1\right]}$ converges to zero and therefore, inside the curly brackets on the right-hand-side of the equation, the first three terms and the seventh term can be dropped; the fifth term becomes negligible compared to the fourth term; and, finally, in the last term, $\operatorname{Iv}\left(P e_{s p}\right)$ can be approximated by $1-1 / P e_{s p}$ [26]. Therefore, equation (7) simplifies to

$$
\frac{\delta_{s p}}{R_{s p}}=\frac{1}{P e_{s p}}
$$


Interestingly, equation (8) indicates that in in the high $P e_{s p}$ limit, $\delta_{s p}$ does not depend on $R_{f}$. Using the definition of $P e_{s p}$, equation (8) can be recast into

$$
\delta_{s p}=\frac{D_{l}}{w_{s p}}
$$

The second interesting limiting case of equation (7) is the high $R_{f}$ limit. In this limit, similar to the high $P e_{s p}$ limit discussed above, $e^{-P e_{s p}\left[\left(R_{f} / R_{s p}\right)-1\right]}$ converges to zero. Therefore, inside the curly brackets, the first three terms and the seventh term can be dropped; the fourth and fifth terms become negligible compared to the sixth term; finally, in the denominator of the term outside the curly brackets, $R_{s p}^{3}$ becomes negligible compared to $R_{f}^{3}$; therefore, equation (7) reduces to

$$
\frac{\delta_{s p}}{R_{s p}}=1-\operatorname{Iv}\left(P e_{s p}\right)
$$

Note that the high $P e_{s p}$ limit of this equation is, as expected, identical to the high $P e_{s p}$ limit of equation (7) (i.e., equation (8)). In the low $P e_{s p}$ limit, one has $\operatorname{Iv}\left(P e_{s p}\right) \rightarrow 0$ [18] and equation (10) reduces to

$$
\delta_{s p}=R_{s p}
$$

\subsubsection{Primary Tip Velocity}

Macroscopic models need to predict the primary tip velocity, $V_{t}$, referred to as the tip velocity hereafter, because the growth of an envelope, at least during the early stages, is mainly driven by the growth of its primary arms. Therefore, the tip velocity, $V_{t}$ can be expected to be one of the 
main, if not the main factor, in determining $w_{s p}$. In addition, $V_{t}$ is required in predicting the primary arm length $l_{t}$ from

$$
\frac{d l_{t}}{d t}=V_{t}
$$

To understand the variations of $V_{t}$ during the quasi-steady growth of an assembly of dendrites, let us first consider two dendrites located at the distance $2 R_{f}$ from each other inside a uniformly undercooled melt, as shown schematically in Figure 2 at (a) an early time and (b) a late time during growth. Due to the symmetry, only half of the dendrites are shown. The profiles of solute concentration in the extra-dendritic liquid are also shown in the figure. Note that the concentration at the tip is equal to the equilibrium concentration $C_{l}^{*}$ and it decreases as one moves away from the tip towards the liquid. At the early time (i.e., Figure 2(a)), this decrease continues until some distance ahead of the tip, where the concentration reaches the initial solute concentration $C_{0}$ and remains constant after that; therefore, the concentration at the symmetry line between the grains $C_{f}$ is equal to $C_{0}: C_{f}=C_{0}$; at the late time (i.e., Figure 2(b)), the decrease continues through the entire liquid region up to the symmetry line between the two grains, where the concentration reaches $C_{f}$, which has a value greater than $C_{0}: C_{f}>C_{0}$.

At the early stage of growth, shown in Figure 2(a), there is a distance between the edges of the solutal boundary layers ahead of the tips and, therefore, the solutal field ahead of one dendrite is not influenced by the presence of the other. In other words, the dendrites are not interacting. This stage of growth is, therefore, referred to as the non-interacting stage. At this stage, the growth of the dendrites is virtually the same as the growth of a single dendrite into an essentially infinite medium. As the dendrites keep growing, the distance between the edges of the boundary layers decreases and at some intermediate time the edges meet. Once that happens, the solutal boundary layer ahead of each of the dendrites starts to get influenced by the presence of the other dendrite. 
In other words, the dendrites start to interact. This stage is called the interacting stage. Next, the variations of $\bar{C}_{e}$ and $C_{f}$ during these two stages and the relationships between them and $C_{0}$ are discussed. During the non-interacting stage, $C_{f}$ remains constant and equal to $\bar{C}_{0}$; furthermore, $C_{f}$ is less than the average solute concentration in the extra-dendritic liquid $\bar{C}_{e}(t)$ : $C_{0}=C_{f}<\bar{C}_{e}(t)$. During the interacting stage, however, $C_{f}$ is greater than $\bar{C}_{0}$ but still less than $\bar{C}_{e}(t): C_{0}<C_{f}<\bar{C}_{e}(t)$. These two relations are important, and will be referred to subsequently when the time variations of $V_{t}$ during these two stages is discussed.

As the primary arm of a dendrite grows, it rejects solute (assuming $k_{0}<1$ ). For growth to continue, the rejected solute needs to be dissipated away from the tip towards the bulk liquid. The balance between the solute flux rejected at the tip and the solute flux diffusing away from the tip determines the tip velocity. The latter flux is proportional to the solute gradient at the tip. During the noninteracting stage of growth, the diffusion field ahead of the tip and therefore the diffusion flux at the tip remain constant. This causes $V_{t}$ to remain constant. During the interacting stage, however, $C_{f}$ increases with time, which makes the solute profiles progressively smoother; therefore, the solute diffusion flux at the tip and consequently $V_{t}$ both decrease with time. Prediction of $V_{t}$ during these stages is discussed next.

In macroscopic models of solidification, the most commonly used relation for predicting $V_{t}$ is the relation proposed by Ivantsov [27]:

$$
\Omega_{t, e f f}=P e_{t} \exp \left(P e_{t}\right) \mathrm{E}_{1}\left(P e_{t}\right)
$$

where $\Omega_{t, \text { eff }}$ is an effective tip undercooling (i.e., the undercooling corresponding to the effective far-field solute concentration, which should not be confused with the solute concentration at the symmetry line between two adjacent grains $C_{f}$ discussed earlier in connection with Figure 2) and 
$P e_{t}=V_{t} R_{t} /\left(2 D_{l}\right)$ is the dendrite tip Péclet number, $R_{t}=\sqrt{d_{0} D_{l} /\left(V_{t} \sigma^{*}\right)}$ is the tip radius, $k_{0}$ is the partition coefficient, $d_{0}$ is the capillary length, $\sigma^{*}$ is the tip selection parameter, and the function $E_{1}(\quad)$ is the exponential integral function. Equation (13) is the exact similarity solution for the solute diffusion field around a paraboloid of revolution during its quasi-steady shapepreserving growth into an infinite medium with uniform and constant far-field undercooling $\Omega_{t, e f f}$ . This equation has been shown to provide accurate predictions of the primary tip velocity $V_{t}$ during the quasi-steady growth of a single dendrite into an essentially infinite medium [28]. For the quasi-steady growth of multiple dendrites, equation (13) can be expected to accurately predict $V_{t}$ during the non-interacting stage. To predict $V_{t}$ during the interacting stage, modifications to this equation have been proposed [29, 30]. These modifications are, however, limited to isothermal dendrites and specific dendritic arrangements and a generally valid relation to predict $V_{t}$ during the interacting stage is still not available. Therefore, similar to the numerous studies in the literature $[5,7,8,18,31]$, in this paper, equation (13) is used to predict $V_{t}$ during both non-interacting and interacting stages.

In using equation (13) to predict $V_{t}$ during the growth of multiple dendrites one should keep in mind that, as depicted in Figure 2 and discussed in the figure discussion, during both the interacting and non-interacting stages, one has $C_{f}<\bar{C}_{e}$; since the effective far-field solute concentration is equal to and less than $C_{f}$ during the non-interacting and interacting stages, respectively, the effective tip undercooling $\Omega_{t, \text { eff }}$ will be always higher than the average undercooling in the extradendritic liquid $\Omega_{e}$, which is defined as

$$
\Omega_{e}=\frac{C_{l}^{*}-\bar{C}_{e}}{\left(1-k_{0}\right) C_{l}^{*}}
$$


In other words, during the entire growth period, one has $\Omega_{e}<\Omega_{t, e f f}$. Therefore, if, in equation (13), $\Omega_{e}$ is used instead of $\Omega_{t, e f f}$, the tip velocity $V_{t}$ will be underpredicted. Using $\Omega_{e}$ in this equation has been, however, a common practice in the literature $[5,7,8]$ because, currently, there are no relations to predict $\Omega_{t, \text { eff }}$. Developing a relation to predict $\Omega_{t, \text { eff }}$ is one of the objectives of this study.

\section{Mesoscopic Envelope Model}

The mesoscopic envelope model used in the present study was originally developed by Delaleau [14] and recently used by Souhar et al. [15] to perform three-dimensional simulations of equiaxed

growth. The reader is referred to these papers for the details of the model and the complete set of equations. In brief, the model approximates the complex dendritic structure with an envelope and a solid fraction field inside the envelope. The normal growth velocity at any point on the envelope is calculated from the local dendrite tip velocity, obtained from an analytical stagnant film model, and the angle between the growing dendrite arm and the envelope normal. The stagnant film model gives the tip local velocity as a function of the undercooling of the liquid in the vicinity of the envelope. The envelope growth and the solute transport in the liquid around the envelope are thus coupled. The liquid inside the envelope and on the envelope surface is assumed to be well-mixed and in equilibrium with the solid while the liquid outside the envelope is generally undercooled. The solid fraction field inside the envelope and the solute concentration field in the extra-dendritic liquid outside the envelope $C_{e}$ are obtained from the numerical solution of a solute conservation equation that is valid both inside and outside the envelope. Hence, the solutal interactions between the growing grains are fully resolved.

\subsection{Mesoscopic Simulations}


The first set of mesoscopic simulations were performed for the isothermal growth of a single grain growing into an essentially infinite domain (Figure 3(a)) and for multiple grains (Figure 3(b)) with high/low grain densities of $R_{f} /\left[D_{l} / V_{I v}\left(\Omega_{0}\right)\right]=4.03 / 6.31$, where $V_{I v}\left(\Omega_{0}\right)$ is the Ivantsov tip velocity (i.e., the velocity predicted by equation (13)) corresponding to the initial undercooling $\Omega_{0}$. Each case was simulated for $\Omega_{0}=0.05$ and 0.15 . For the multiple grain cases, the grains were arranged periodically in a BCC lattice, with the primary arms growing along the axes (Figure 3(b)).

In Figure (4), an example of the mesoscopic simulation results is shown. The figure, which is for the multiple grain case with the low undercooling $\left(\Omega_{0}=0.05\right)$ and high grain density ( $\left.R_{f} /\left[D_{l} / V_{I v}\left(\Omega_{0}\right)\right]=4.03\right)$, shows the solid fraction, $g_{s}$, and the solute concentration in the extradendritic liquid $C_{e}$, plotted in the interior and exterior of the envelopes, respectively, at different times. The dimensionless time, $\tau$, is scaled by the time needed for a steady-state tip to advance by one diffusion length: $V_{I v}\left(\Omega_{0}\right) /\left(D_{l} / V_{I v}\left(\Omega_{0}\right)\right)$. It can be seen that, as expected (see the discussion below Equation (4)), the envelope is initially spherical (see Figure 4(a)), but it gradually becomes dendritic during growth. It can also be seen that during the growth, the envelopes reject solute to the extra-dendritic liquid and, therefore, $C_{e}$ increases. At the early times (i.e., $\tau<0.37$ ), this increase is limited to a relatively small distance ahead of the envelopes; therefore, $C_{e}$ further away from the envelopes is still at the initial value of $0.5 \mathrm{wt}$. pct.. At the later times (i.e., $\tau>2.23$ ), $C_{e}$ everywhere in the domain has become greater than 0.5 . Finally, at $\tau=5.94, C_{e}$ everywhere has reached the equilibrium solute concentration $C_{l}^{*}=0.523$; the undercooling has fully vanished and the growth has ended.

Another example of the mesoscopic simulation results is shown in Figure 5. The figure, which, similar to Figure 4, is for the multiple grain case with the low undercooling and high grain density, shows the final envelope shape and the profiles of solute concentration in liquid along the line connecting the primary arms of two dendrites growing towards each other. Different curves show 
the profiles at different times. From the plot it can be seen that at $\tau=2.6$ the solutal fields ahead of the grain envelopes overlap and, therefore, the grains start interacting.

\subsection{Upscaling Mesoscopic Results}

To upscale the mesoscopic simulations results, they were averaged over the volume of the REV. For example, at any time during growth, the solute concentration field in the extra-dendritic liquid was averaged over the volume of the REV to give the value of $\bar{C}_{e}$ at that time. In Figure 6, the upscaled mesoscopic results are plotted as a function of the non-dimensional time defined as $\tau=t V_{l v}^{2}\left(\Omega_{0}\right) / D_{l}$. Results for a single grain are shown as black curves and for multiple grains with high and low grain density as red and blue curves, respectively. Results for $\Omega_{0}=0.05$ and 0.15 are plotted as solid and dashed curves, respectively.

Figure 6(a) shows the envelope volume fraction $g_{e n v}$. Squares in the figure represent the start of the second stage of growth and the definitions of the first and second stages will become clear subsequently, when Figure 6(g) is discussed. Figure 6(b) shows the non-dimensional average undercooling in the extra-dendritic liquid $\Omega_{e} / \Omega_{0}$, where $\Omega_{e}$ was calculated from equation (14). Figure 6(c) and Figure 6(d), a close-up of 6(c) around $\tau=5$, show the sphericity, which was calculated using equation (3) after calculating $S_{e n v}$ and $S_{s p}$ as follows: $S_{e n v}$ was measured directly from the simulated envelope shape, and $S_{s p}$ was calculated from equation (5), after computing $R_{s p}$ from an equation that is derived subsequently in connection with Figure 6(h). Figure 6(e) shows the non-dimensional primary arm length $l_{t} /\left[D_{l} / V_{I_{v}}\left(\Omega_{0}\right)\right]$, where $l_{t}$ was measured directly from the simulated envelope shape. Figure 6(f) shows the non-dimensional tip velocity $V_{t} / V_{l v}\left(\Omega_{0}\right)$, where $V_{t}$ was calculated from equation (12). Figure $6(\mathrm{~g})$ shows the scaled primary arm length $l_{t}^{*}$ defined as 


$$
l_{t}^{*}=\frac{l_{t}}{l_{\text {diff }}}
$$

where $l_{\text {diff }}$ is the instantaneous diffusion length ahead of the primary tip, which is defined as

$$
l_{\text {diff }}=\frac{D_{l}}{V_{t}}
$$

Figure 6(h) shows the non-dimensional sphere radius $R_{s p} /\left[D_{l} / V_{I v}\left(\Omega_{0}\right)\right]$. The sphere radius, $R_{s p}$, is calculated using an equation that relates $R_{s p}$ to the final grain radus $R_{f}$, to the envelope fraction, $g_{\text {env }}$, and to the effective number of grains inside the REV, $n$. In what follows, this equation is first derived using a formal procedure, to show that the equation is the integrated form of equation (1). Next, it is discussed that the equation can be written directly from the definition of the sphere. The formal derivation of the equation starts with first substituting the right-hand side of equation (1) using $S_{e n v} w_{e n v}=S_{s p} w_{s p}$ (see the discussion above equation (4)); then, $S_{s p}$ and $w_{s p}$ are substituted from equations (5) and (6), respectively, to give

$$
\frac{1}{g_{e n v}} \frac{d g_{e n v}}{d t} d t=\frac{3}{R_{s p}} \frac{d R_{s p}}{d t}
$$

Next, the definite integrals of both sides of this equation are taken from time zero to time $t$ to give

$$
\frac{g_{e n v}}{g_{e n v}(t=0)}=\left\lceil\frac{R_{s p}}{R_{s p}(t=0)}\right]^{3}
$$

In this equation, $g_{e n v}(t=0)$ and $R_{s p}(t=0)$ are the envelope fraction and sphere radius corresponding to the initial spherical seeds. Since the initial seeds have the same size, $g_{e n v}(t=0)$ and $R_{s p}(t=0)$ can be related as $R_{s p}(t=0)=2[3 /(4 \pi n)]^{1 / 3} R_{f} g_{e n v}^{1 / 3}(t=0)$, where $n$ is the effective 
number of grains inside the REV, which is equal to unity for a single grain and two for multiple grains in the BCC arrangement. Substituting this equation into equation (18) gives

$$
R_{s p}=2\left(\frac{3}{4 \pi n}\right)^{1 / 3} R_{f} g_{e n v}^{1 / 3}
$$

Note that this equation has a simple physical meaning: it indicates that, as expected, at any time during growth, the total volume of the spheres (i.e., $n \times 4 \pi R_{s p}^{3} / 3$ ) is equal to the total volume of the envelopes (i.e., $8 R_{f}^{3} g_{e n v}$ ). In fact, one can write this equation directly from the definition of the sphere. Here, however, the more formal procedure to derive it is provided to show that equation (19) is indeed the integrated form of equation (1).

Figure 6(i) shows the non-dimensional sphere velocity $w_{s p} / V_{I v}\left(\Omega_{0}\right)$, where $w_{s p}$ was calculated from equation (6). Figure 6(j) shows the non-dimensional average diffusion length around the envelopes $\delta_{e n v} /\left[D_{l} / V_{l v}\left(\Omega_{0}\right)\right]$, where $\delta_{e n v}$ was determined from equation (2) by solving this equation for $\delta_{e n v}$, using the upscaled mesoscopic values for all the other quantities. Finally, Figure 7 shows the comparison between the mesoscopic primary tip velocities and the Ivantsov tip velocities corresponding to $\Omega_{e}$. Next, the important observations that can be made from these plots are discussed.

From Figures 6(a) and 6(b) it can be seen that for a single grain $g_{e n v}$ and $\Omega_{e}$ remain close to zero and $\Omega_{0}$, respectively, during the entire growth. This is because for the single grain cases the size of the simulation domain was chosen to be large enough to remain much larger than the envelope size during the entire growth. For the multigrain cases, however, the envelope fraction increase relatively fast initially because $\Omega_{e}$, which is the driving force for growth, is relatively high; as $\Omega_{e}$ decreases, due to the solute rejection from the envelopes to the extra-dendritic liquid, the rate of 
increase in $g_{e n v}$ decreases. Finally, when the undercooling is fully consumed (at $\tau$ about 4 and 9 for the high and low grain density cases, respectively) $g_{e n v}$ ceases to increase further and growth ends.

From Figures 6(c) and 6(d) it can be seen that, as expected, the initial value of $\psi$ is equal to unity and as the envelope becomes progressively more dendritic with growth, $\psi$ decreases. For a single grain, this decrease continues until $\tau=40$. At this time, we stopped the simulations because the diffusion field around the envelope started to interact with the boundaries of the simulation domain. For the multigrain cases, however, after a relatively small initial decrease (of about 0.1 for the high grain density cases and 0.2 for the low grain density cases) $\psi$ stops to decrease further and then remains constant.

From Figure 6(f) it can be seen that at an early stage of growth ( $\tau$ less than two) we have $1<V_{t} / V_{I v}\left(\Omega_{0}\right)$ : the mesoscopic tip velocities $V_{t}$ are greater than the Ivantsov tip velocities corresponding to the initial undercooling $V_{I v}\left(\Omega_{0}\right)$. This is because of the presence of an initial transient stage in the mesoscopic simulations, where the $C_{e}$ field is transitioning from the initial value of $C_{e}=C_{0}$ (see Figure 6(a)) to the quasi-steady values. During this stage, the solutal gradient ahead of the tip and therefore the tip velocity is greater than the quasi-steady values predicted by equation (13).

At the end of the initial transient stage ( $\tau$ about 2), the quasi-steady stage starts. During this stage, $V_{t}$ for a single grain (i.e. the black curves) remains, as expected, constant, but at a value that is slightly (about 10 percent) lower than the Ivantsov tip velocity corresponding to the initial undercooling $\Omega_{0}$. This minor underprediction of the tip velocities by the mesoscopic model is of no significant consequence and should not distract; however, for the sake of completeness, the reason for it is explained next. As already discussed in detail by Steinbach et al. [1,2] and Souhar 
et al. $[15,32]$, in the mesoscopic model, the predicted tip velocities depend on a parameter in the model known as the stagnant film thickness $\delta_{f}$. For the high values of $\delta_{f}$ (i.e. $\delta_{f}>3 l_{\text {diff }}[15$, 32]) the mesoscopic tip velocity for a single grain will be equal to the Ivantsov tip velocity. However, with such a high value of $\delta_{f}$, the predicted grain envelope shapes will be unrealistic (compared to the experimentally observed ones [16]). Therefore, to have relatively accurate predictions for both $V_{t}$ and the envelope shape, a compromising intermediate value for $\delta_{f}$ needs to be chosen. As a result of this compromise, the quasi-steady mesoscopic tip velocities are slightly lower than the Ivantsov tip velocities.

The tip velocity $V_{t}$ for the multiple grain cases starts to rapidly decrease at some intermediate time (about $\tau=2$ for the high grain density cases and 4.5 for the low grain density cases). This rapid decrease is physically important and indicates that the tips are solutally interacting.

From Figure 6(g) it can be seen that $l_{t}^{*}$, which was defined in equation (15), for single grain cases increases with time during the entire growth period. For the multigrain cases, however, $l_{t}^{*}$ increases with time initially, but, at some intermediate time which is denoted by the squares in the figure, $l_{t}^{*}$ starts to decrease with time and eventually reaches zero (since $l_{\text {diff }} \rightarrow \infty$ as the result of $V_{t} \rightarrow 0$ ). Therefore, the entire growth period can be divided into two stages: the first stage, where $d l_{t}^{*} / d t>0$ , and the second stage, where $d l_{t}^{*} / d t \leq 0$. These two stages should not be confused with the noninteracting and interacting stages discussed in connection with Figure 2. The interacting and noninteracting stages concerned the growth of the primary arms, while the first and second stages introduced here concern the average growth kinetics of the envelopes. It will be shown below that the first and second stages can be referred to as variable-sphericity and constant-sphericity stages, respectively. Dividing the entire growth period into variable-sphericity and constant-sphericity stages based on the sign of $d l_{t}^{*}$ is an important premise that is proposed in this study and will be used in section 4 , where the constitutive relations are developed. 
Variations of $l_{t}^{*}$ during these two stages can be understood by following the variations of $l_{t}$ and $V_{t}$, shown in Figures 6(e) and 6(f), respectively, and focusing on how the nominator and denominator of equation (15) change with time. During the first stage, $V_{t}$ is relatively high (i.e., greater than $\left.0.8 \times V_{I v}\left(\Omega_{0}\right)\right)$ and therefore, $l_{t}$, which appears in the nominator of equation (15), increases relatively fast; this causes $l_{t}^{*}$ to increase with time during the first stage. When the second stage starts, $V_{t}$ has an intermediate value and, more importantly, is decreasing fast. Therefore, unlike in the first stage, the increase in $l_{t}$ is not fast anymore and becomes insignificant compared to the fast increase in $l_{\text {diff }}$, which appears in the denominator of equation (15); this causes, $l_{t}^{*}$ to decrease with time during the second stage.

There is one last interesting point about Figure 6(c) that can be discussed now because the first and second stages of growth were defined. It can be seen from this figure that the variations of the sphericity during the second stage of growth (i.e., the right-hand side of the squares) are negligible compared to these variations during the first stage of growth and, therefore, the sphericity can be assumed to be constant during the second stage of growth. Consequently, in the rest of the paper, the first and second stages of growth are referred to as variable-sphericity and constant-sphericity stages, respectively.

Figure 6(i) shows the time variations of the non-dimensional sphere velocity. From the figure, it can be seen that during the variable-sphericity stage of growth (i.e., the left-hand side of the squares), the multigrain curves collapse on the single grain curves. This indicates that during the variable-sphericity stage of growth, $w_{s p}$ for multigrain and single grain cases can be expected to be predicted by the same relation. When the constant-sphericity stage starts, however, the multigrain curves cease to collapse on the single grain curves, and they start decreasing relatively 
rapidly. This indicates that $w_{s p}$ during the constant-sphericity stage of growth needs to be predicted from a separate relation.

Comparing the time variations of $V_{t}$, shown in Figure 6(f), and time variations of $w_{s p}$, shown in Figure 6(i), reveals another interesting observation. Focusing first on the low grain density cases (the blue curves), one can see that at $\tau \square 8, V_{t}$ is zero: the primary tips have fully stopped. At the same time, however, $w_{s p}$ is still greater than zero: the envelopes are still growing. This indicates that growth continues (at least until $\tau \square 10$ ) even after the primary tips stop. A similar trend is observed for the low grain density curves. Growth of an envelope after the primary tips stop is due to the growth of the secondary arms.

In Figure 7, the mesoscopic primary tip velocities (the thin curves) are compared with the Ivantsov tip velocities, predicted using equation (13) with $\Omega_{t, \text { eff }}=\Omega_{e}$ (the thick curves). Data are shown only for the multigrain cases. One can see from the figure that, as expected (see the discussion below equation (14)), setting $\Omega_{t, e f f}=\Omega_{e}$ in the Ivantsov solution significantly underpredicts the tip velocities.

Finally, this section is ended by summarizing the important observations that can be made from the upscaled mesoscopic results: 1) for the multiple grain cases, the entire growth period can be divided into the variable-sphericity and constant-sphericity stages and these two stages correspond to the positive and negative values of $d l_{t}^{*}$, respectively; 2) for the single grain cases, the growth takes place entirely in the first stage; 3) setting $\Omega_{t, e f f}=\Omega_{e}$ in the Ivantsov relation will significantly underpredict the tip velocities.

\section{Constitutive Relations}




\subsection{Postulates}

It is postulated that during the variable-sphericity stage of growth, $\psi$ is a function of $l_{t} / R_{s p}$ only and during the constant-sphericity stage of growth, $\psi$ is, obviously, constant.

$$
\begin{aligned}
& d l_{t}^{*}>0 \quad \rightarrow \quad \psi=\psi\left(\frac{l_{t}}{R_{s p}}\right) \\
& d l_{t}^{*}<=0 \quad \rightarrow \quad d \psi=0
\end{aligned}
$$

It is known from the literature [27] that the shape of a dendrite depends on the surface tension anisotropy; therefore, one might wonder why such a dependence is not introduced in equation (20). This is because in this equation (and through the entire paper) $\psi$ is the sphericity of the dendrite envelope and, therefore, depends on the envelope shape (which shall not be confused with the dendrite shape). From what is available in the literature, it is not clear whether the envelope shape depends on the surface tension anisotropy or not. What is known from the literature is that the envelope shape, and therefore, the sphericity predicted by the mesoscopic model, has been validated against experiments of equiaxed solidification of SCN-acetone $[1,15]$ and of directional solidification of Al-Cu [14]. Since, as will be shown in section 5, the mesoscopic values of $\psi$ are accurately predicted by taking the sphericity as a function of $l_{t} / R_{s p}$ only, introducing surface tension anisotropy effects in equation (20) does not seem to be necessary.

To develop a relation for $w_{s p}$, one first needs to recognize that the average growth kinetics, and therefore $w_{s p}$, are in general determined by the growth of both the primary and the secondary arms. At early stages of growth, the primary arms grow much faster than the secondary arms. Therefore, their velocity can be expected to be the main factor in determining $w_{s p}$. As the growth continues, the primary arms slow down and finally stop, but the secondary arms and therefore the sphere continue to grow, until the undercooling in the extra-dendritic liquid fully vanishes (i.e., the 
average undercooling in the extra-dendritic liquid reaches zero). In other words, at some intermediate time during growth, the main mechanism that drives the envelope growth, and thus determines $w_{s p}$, transitions from the primary tip velocity to the average undercooling of the extradendritic liquid. This transition and the time at which it occurs need to be properly taken into account in developing the relation for $w_{s p}$. In this paper, it is first postulated that the transition occurs when the constant-sphericity stage of growth starts. The postulates to determine $w_{s p}$ during the variable-sphericity and constant-sphericity stages are discussed next.

During the variable-sphericity stage, $w_{s p}$ is assumed to scale with the primary tip velocity $V_{t}$. It should be noted that $w_{s p} / V_{t}$ cannot be taken as constant because the shape of the envelope changes significantly during the variable-sphericity stage. In the macroscopic model, the envelope shape is represented solely by the envelope sphericity $\psi$. In other words, $\psi$ is assumed to contain all the geometrical information about the envelope shape. Since $\psi$ itself is postulated to be a function of $l_{t} / R_{s p}$ only (see equation (20)), $w_{s p} / V_{t}$ is similarly postulated to be a function of $l_{t} / R_{s p}$ only. During the constant-sphericity stage, $w_{s p}$ is assumed to scale with $w_{s p}\left(t_{s}\right)$, where $t_{s}$ is the time at the start of the constant-sphericity stage, and the ratio $w_{s p} / w_{s p}\left(t_{s}\right)$ is postulated to be a function of the scaled Ivantsov velocity corresponding to $\Omega_{e}, V_{I v}\left(\Omega_{e}\right) / V_{I v}\left[\Omega_{e}\left(t_{s}\right)\right]$, only. The above three postulates can be expressed mathematically as

$$
\begin{aligned}
& d l_{t}^{*}>0 \rightarrow \frac{w_{s p}}{V_{t}}=\frac{w_{s p}}{V_{t}}\left(\frac{l_{t}}{R_{s p}}\right) \\
& d l_{t}^{*} \leq 0 \rightarrow \frac{w_{s p}}{w_{s p}\left(t_{s}\right)}=\frac{w_{s p}}{w_{s p}\left(t_{s}\right)}\left\{\frac{V_{I v}\left(\Omega_{e}\right)}{V_{I v}\left[\Omega_{e}\left(t_{s}\right)\right]}\right\}
\end{aligned}
$$


Through the entire growth period, $\Omega_{t, e f f}$ is assumed to scale with $\Omega_{e}$, and the ratio $\Omega_{t, e f f} / \Omega_{e}$ is assumed to be a function of the scaled length of the free liquid region ahead of the primary tip up to the symmetry line between two adjacent grains $l_{l}^{*}=R_{f}^{*}-l_{t}^{*}$, where $R_{f}^{*}=R_{f} / l_{\text {diff }}$ :

$$
\frac{\Omega_{t, e f f}}{\Omega_{e}}=\frac{\Omega_{t, e f f}}{\Omega_{e}}\left(l_{l}^{*}\right)
$$

The envelope diffusion length, $\delta_{e n v}$, is assumed to scale with the sphere diffusion length, $\delta_{s p}$ (Eq. (7)), and the ratio $\delta_{e n v} / \delta_{s p}$ is assumed to be a function of sphericity only:

$$
\frac{\delta_{e n v}}{\delta_{s p}}=\frac{\delta_{e n v}}{\delta_{s p}}(\psi)
$$

Note that $\delta_{e n v} / \delta_{s p}$ could have been formulated as a function of $l_{t} / R_{s p}$ instead of $\psi$, because $\psi$ is a function of $l_{t} / R_{s p}$ only (see equation (18)) but here it is formulated as a function of the envelope sphericity $\psi$ to better illustrate that the ratio of the envelope diffusion length to the sphere diffusion length is a function of the envelope geometry, which is represented by the envelope sphericity.

\subsection{Fitting Functions}

In this section, the upscale mesoscopic results, presented in section 3.2, are used to plot the lefthand-side of equations (20) to (23) as a function of the independent variable on the right-handside. The constitutive relations are then developed by curve fitting these plots. In the following figures, mesoscopic results for a single grain are shown as black curves and for multiple grains with high and low grain density as red and blue curves, respectively. Results for $\Omega_{0}=0.05$ and 
0.15 are plotted as solid and dashed curves, respectively; the green curves depict our curve fits and the squares show the start of the constant-sphericity stage of growth.

In Figure 8, the sphericity $\psi$ is plotted as a function of the ratio of the primary dendrite arm length to the sphere radius $l_{t} / R_{s p}$. It can be seen that for a single grain, the mesoscopic simulation results for the two different initial undercoolings $\Omega_{0}$ collapse onto a single curve. This indicates that the sphericity is indeed a function of $l_{t} / R_{s p}$ only. The multigrain data in the plot fall on the same curve as the single grain data during the variable-sphericity stage of growth. However, when the constant-sphericity stage starts, the multigrain data start to deviate slightly from the sphericity curve for a single grain. The variation of $\psi$ during this stage are, however, extremely small and are disregarded. The final fit of the sphericity data for both the single grain and the multigrain cases is then given by:

$$
\begin{aligned}
& d l_{t}^{*}>0 \quad \rightarrow \quad \psi=1-\frac{6.34}{6.02+\frac{8.08}{\left(l_{t} / R_{s p}-1\right)^{1.93}}} \\
& d l_{t}^{*}<=0 \quad \rightarrow \quad d \psi=0
\end{aligned}
$$

This equality can be understood as follows. Initially (i.e., $\tau=0$ ), the envelope is spherical and $l_{t} / R_{s p}$ is equal to unity; therefore the denominator on the right-hand side will be large, which will make the sphericity become equal to unity. During growth, as the envelope shape transitions from a spherical to a dendritic, $l_{t} / R_{s p}$ increases; the second term on the right-hand-side increases, and therefore $\psi$ decreases.

In Figure 9(a), $w_{s p} / V_{t}$ during the variable-sphericity stage of growth is plotted as a function of $l_{t} / R_{s p}$. It can be seen that $w_{s p} / V_{t}$ decreases monotonically as $l_{t} / R_{s p}$ increases. Since the single grain and multigrain data for the two different initial undercoolings $\Omega_{0}$ collapse, $w_{s p} / V_{t}$ during 
the variable-sphericity stage of growth is indeed a function of $l_{t} / R_{s p}$ only, and can be fit by $w_{s p} / V_{t}=0.80-0.78\left[1-1 /\left(l_{t} / R_{s p}\right)\right]^{0.85}$. In Figure 9(b), $w_{s p} / w_{s p}\left(t_{s}\right)$ is plotted as a function of $V_{I v}\left(\Omega_{e}\right) / V_{I v}\left[\Omega_{e}\left(t_{s}\right)\right]$. Single grain data cannot be included in this plot because, as discussed in connection with Figure 6(g), for a single grain growth takes place solely at the variable-sphericity stage. One can see that the multigrain data for the two different initial undercoolings $\Omega_{0}$ collapse onto a single curve. This indicates that $w_{s p} / w_{s p}\left(\tau_{s}\right)$ is indeed a function of $V_{I v}\left(\Omega_{e}\right) / V_{I v}\left[\Omega_{e}\left(\tau_{s}\right)\right]$ only. The final fit for $w_{s p} / w_{s p}\left(\tau_{s}\right)$ is then given by $w_{s p} / w_{s p}\left(t_{s}\right)=\left\{V_{I v}\left(\Omega_{e}\right) / V_{I v}\left[\Omega_{e}\left(t_{s}\right)\right]\right\}^{0.50}$. Summarizing the fits proposed in Figures 9(a) and 9(b), we get

$$
\begin{aligned}
& d l_{t}^{*}>0 \rightarrow \frac{w_{s p}}{V_{t}}=0.80-0.78\left(1-\frac{1}{l_{t} / R_{s p}}\right)^{0.85} \\
& d l_{t}^{*} \leq 0 \rightarrow \frac{w_{s p}}{w_{s p}\left(t_{s}\right)}=\left\{\frac{V_{I v}\left(\Omega_{e}\right)}{V_{I v}\left[\Omega_{e}\left(t_{s}\right)\right]}\right\}^{0.50}
\end{aligned}
$$

In Figure 10, the scaled effective far-field undercooling $\Omega_{t, \text { eff }} / \Omega_{e}$ is plotted as a function of $l_{l}^{*}$; $\Omega_{t, e f f}$ was calculated from Equation (13), together with the relation for the tip radius (see the discussion below Equation (13)), using the mesoscopic values for $V_{t}$. Note that $\Omega_{t, e f f}$ is an effective undercooling that is to be used in the Ivantsov relation (Eq. (13)) in order to obtain accurate tip velocities for the primary tips of interacting dendrites. Data is shown only during the variable-sphericity stage of growth because the curves for the different mesoscopic cases did not collapse during the constant-sphericity stage of growth. This, however, should not distract because, as will become clear at the end of this section, in the macroscopic model, the calculation of $V_{t}$ and therefore $\Omega_{t, \text { eff }}$ are not required during the constant-sphericity stage. Variations of $\Omega_{t, e f f} / \Omega_{e}$ with $l_{l}^{*}$ can be best understood by first focusing on the data for the high undercooling low grain density case (i.e., the dashed blue curve). Initially (i.e., at $\tau=0$ ), $l_{l}^{*}$ has its highest value (about six) and $\Omega_{t, e f f}$ is slightly greater than $\Omega_{e}$, which is equal to $\Omega_{0}$; this is because, as shown in Figure 6(f) 
and due to the presence of the initial transient stage, $V_{t}$ is initially greater than $V_{I v}\left(\Omega_{0}\right)$. During growth, $l_{t}$ increases and therefore $l_{l}^{*}$ decreases. For $l_{l}^{*}>2, \Omega_{t, e f f} / \Omega_{e}$ remains almost constant because the rate of decrease in $\Omega_{t, e f f}$ and $\Omega_{e}$ are almost the same; however, at $l_{l}^{*}$ about two, $\Omega_{t, e f f} / \Omega_{e}$ starts to increase because the rate of decrease in $\Omega_{e}$ starts to become greater than the rate of decrease in $\Omega_{t, e f f}$. The curves for the other mesoscopic cases behave in a similar fashion and, despite the minor spread between them, they can be fit by a single curve given by

$$
\frac{\Omega_{t, e f f}}{\Omega_{e}}=\frac{1}{0.97-\frac{0.28}{1+\left(l_{l}^{*} / 1.3\right)^{1.95}}}
$$

There are two more points about Figure 10 and equation (26) that need to be discussed before pursuing. First, focusing on the blue curves in the figure, it can be seen that they do not collapse fully and have a minor spread. This minor spread causes imperfections in the fit for $\Omega_{f} / \Omega_{e}$ and is attributed to the presence of an initial transient stage in the mesoscopic simulations. The collapse between these curves cannot reasonably be expected to be better because, as was shown in Figure 6(f), the curves representing $V_{t}$ for the mesoscopic cases with different initial undercoolings did not collapse during the initial transient stage. Similarly, the collapse between the red curves in Figure 10 cannot be expected to be better. Second, the first term in the denominator of the righthand-side is chosen to be slightly less than unity. In this way, for free growth of a single grain and at the early stages of multigrain growth, where the second term in the denominator is almost zero because $l_{l}{ }^{*}$ is high, $\Omega_{t, e f f}$ becomes slightly higher than $\Omega_{e}\left(\Omega_{t, e f f}=1.031 \Omega_{e}\right)$. Therefore, initially (i.e., at $\tau=0$ ), the predicted tip velocity $V_{t}$ will be higher than $V_{I v}\left(\Omega_{0}\right)$. This means that the macroscopic model can predict the initial transient effects on the tip velocity. However, since the stagnant-film model used to calculate the tip velocity in the mesoscopic model is based on the assumption of a steady-state diffusion field within the stagnant film, the tip velocities predicted by the macroscopic model during the initial transient stage can only be expected to be approximate. 
In Figure 11, $\delta_{e n v} / \delta_{s p}$ for the single grain cases is plotted as a function of $\psi$. It can be seen that as $\psi$ decreases during growth, $\delta_{e n v} / \delta_{s p}$ increases monotonically above unity. This indicates that the diffusion length around a complex shaped dendritic envelope is greater than the diffusion length for the sphere. Since the data for the two different initial undercoolings collapse, $\delta_{e n v} / \delta_{s p}$ is indeed a function of $\psi$ only. A curve fit to the data for the single grain is given by:

$$
\frac{\delta_{e n v}}{\delta_{s p}}=1+1.3 \times(1-\psi)^{2.5}
$$

In summary, the macroscopic model consists of equations (1) to (7), (12) to (16), and (24) to (27). It requires two inputs: the initial undercooling $\Omega_{0}$ and the final grain radius $R_{f}$. Also, note that $l_{t}$ and $V_{t}$ appear only in the first relation of equations (24) and (25), respectively. Therefore, the model needs to calculate $V_{t}$ during the variable-sphericity stage only.

\section{Comparing the Macroscopic Predictions with the Upscaled Mesoscopic Results}

In this section, the constitutive relations are verified by comparing the predictions of the macroscopic model against the upscaled mesoscopic results. It is pointed out that the individual constitutive relations that were each separately fitted to the upscaled mesoscopic simulations are now used in conjunction in a closed macroscopic model. The comparisons are first made for the isothermal mesoscopic cases, presented in section 3.2, and used in section 4 to develop the constitutive relations. Next, to provide further confidence in the constitutive relations, comparisons are made against new upscaled mesoscopic results that are representative for conditions in solidification processing of metal alloys. 


\subsection{Isothermal Cases}

Figures 12 and 13 show the comparisons between the predictions of the macroscopic model for different quantities (the thick curves) with the corresponding upscaled mesoscopic results (the thin curves) for the four isothermal cases. Figure 12 shows the comparisons for the low grain density cases with the low/high undercoolings (the solid/dashed curves) and Figure 13 shows the similar comparison for the high grain density cases. For all the four cases, the overall agreements between the macroscopic predictions and the upscaled mesoscopic results are good and, as is discussed next, cannot realistically be expected to be much better.

In Figures 12(e) and 13(e) the tip velocities predicted by the macroscopic model are compared with the mesoscopic tip velocities. Note that, as discussed at the end of section 4 , in the macroscopic model the tip velocities need to be predicted only during the variable-sphericity stage. Plotting the tip velocities during the constant-sphericity stage for the isothermal cases is only because, for these cases, the mesoscopic and macroscopic predictions of the tip velocity were in good agreement. It can be seen that, during the entire growth period, the predicted tip velocities agree reasonably well with the mesoscopic tip velocities. Contrasting this agreement with the vast difference that was observed in Figure 7 between the mesoscopic tip velocities and the Ivantsov velocities corresponding to $\Omega_{e}$, one can easily acknowledge that the tip velocities predicted by our model (i.e., the Ivantsov velocities corresponding to $\Omega_{t, \text { eff }}$ ) are, as expected, significantly more accurate (when compared against the mesoscopic tip velocities) than the Ivantsov velocities corresponding to $\Omega_{e}$.

The minor difference between the macroscopic and mesoscopic tip velocities is attributed to the presence of the initial transient stage in the mesoscopic simulations, which, as discussed in connection with Figure 10, causes imperfections in our fit for $\Omega_{t, \text { eff }} / \Omega_{e}$. 
Before proceeding, it is necessary to iterate that the constitutive relations developed in this paper are based only on the upscaled mesoscopic results for a single grain growing into an essentially infinite medium as well as multiple grains with the periodic arrangement. In reality, however, grains can have a random arrangement. Therefore, a necessary future work is to examine the agreement between the predictions of the macroscopic model and the upscaled mesoscopic results for multiple grains with a random arrangement. If this agreement is found to be not as good as the agreement that was achieved in Figures 12 and 13, then the constitutive relations will need to be improved. We, however, expect that the predictions of the relation for $\psi$ (i.e., equation (24)), and the relation for $w_{s p}$ during the variable-sphericity stage of growth (i.e., the first equality in equation (25)), will be still in a good agreement with the corresponding mesoscopic results for multiple grains with random arrangment. This is because, for $\psi$ and $w_{s p}$ during the variable-sphericity stage, the mesoscopic data for multiple grains with BCC arrangement collapsed on the data for a single grain (see Figure (8) and 9(a)); therefore, it can be expected that data for multiple grains with random arrangement will also collapse on the single grain data. As a result, it is expected that the fit in Figure 8, and therefore the relation for $\psi$, and also the fit in Figure 9(a), and therefore the relation for $w_{s p}$ during the variable-sphericity stage of growth, will remain unchanged even if mesoscopic data for multiple grains with random arrangement is added to Figure 8 and Figure 9(a). The agreements between the predictions of the relations for $w_{s p}$ during the constant-sphericity stage of growth, $\Omega_{t, e f f}$ and $\delta_{e n v}$ with the corresponding mesoscopic values might not be as good as the agreement observed in Figures 12 and 13. If that turns out to be the case, these relations will need to be improved, and this is a necessary future work.

\subsection{Recalescence Cases}

To further verify the constitutive relations, new mesoscopic simulations were performed and the predictions of the macroscopic model were compared with the upscaled mesoscopic results. These 
new cases are inspired by the experiments of Rappaz and Thevoz [22], which involve solidification of Al-7 wt. pct. Si in a small unit cell, with uniform temperature, under external cooling. These cases are called the recalescence cases because, as is shown below, a temperature recalescence was observed in the cooling curves. To simulate these cases with the macroscopic model, this model needs to be supplemented by three additional equations that are discussed next.

The equation for the energy conservation reads

$$
c_{p} \frac{\partial T}{\partial t}=L_{f} \frac{\partial g_{s}}{\partial t}+\dot{q}
$$

where $c_{p}, L_{f}$, and $\dot{q}$ are the specific heat capacity, latent heat, and heat extraction rate, respectively. To predict the solid fractions, the solute concentration in the inter-dendritic liquid (i.e., the liquid inside the envelopes) was first calculated from [33]:

$$
g_{l} \frac{\partial \bar{C}_{d}}{\partial t}=\left(1-k_{0}\right) \bar{C}_{d} \frac{\partial g_{s}}{\partial t}-\frac{\partial}{\partial t}\left[g_{e}\left(\bar{C}_{e}-\bar{C}_{d}\right)\right]
$$

where $g_{l}=1-g_{s}$ is the liquid fraction. Then, since the inter-dendritic liquid is not undercooled, the solid fraction can be calculated by equating the inter-dendritic liquid solute concentration with the equilibrium solute concentration $C_{l}^{*}$ :

$$
\text { For } T<T_{l i q}\left(\bar{C}_{e}\right)-\Delta T_{n u c}: \bar{C}_{d}=C_{l}^{*}
$$

where $\Delta T_{n u c}$ is the nucleation undercooling and $C_{l}^{*}$ is calculated from the liquidus line of the phase diagram 


$$
C_{l}^{*}=\frac{T-T_{f}}{m_{l}}
$$

where $m_{l}$ is the slope of the liquidus line and $T_{f}$ is the melting point of the pure material. Note that equation (30) assumes that nucleation occurs instantaneously at the nucleation temperature.

Similar to the isothermal cases, the recalescence cases correspond to the growth of multiple grains, in a BCC arrangement, with low/intermediate/high grain densities (i.e., $R_{f}=0.8 / 1.6 / 2.4 \mathrm{~mm}$ ). In the simulations, the properties were taken from Rappaz and Thevoz [22]; the initial temperature was set equal to the liquidus temperature (i.e., no initial undercooling), and the nucleation undercooling was set to zero.

Figures (14) through (17) show the comparisons between the macroscopic predictions of the different quantities (the blue curves) with the corresponding upscaled mesoscopic results (the red curves) for the recalescence cases with low/intermediate/high grain densities. For the recalescence cases, the tip velocities are shown only during the variable-sphericity stage of growth because the mesoscopic and macroscopic predictions of $V_{t}$ did not agree during the constant-sphericity stage. This, however, should not distract because, as discussed earlier, in the macroscopic model, calculation of $V_{t}$ are not required during the constant-sphericity stage. From the plots it can be seen that the overall agreement between the macroscopic and mesoscopic predictions of the different quantities is good. For example, the recalescence observed in the mesoscopic simulations is predicted to within better than $0.5 \mathrm{~K}$ accuracy and the mesoscopic and macroscopic solid fractions are in an almost excellent agreement. Note that the existence of a minor difference between the macroscopic and mesoscopic temperatures while the solid fractions are in excellent agreement, shows extremely high sensitivity of the system (See equation (28) and note that $c_{p}$, 
$L_{f}$, and $\dot{q}$ are constant). Predicting temperatures with accuracy of $0.5 \mathrm{~K}$ for such a sensitive system is a remarkable achievement of the new constitutive relations.

Finally, it is useful to mention that, for the recalescence cases, the macroscopic and mesoscopic values of $V_{t}$ are initially in an excellent agreement. This is because these cases do not have an initial transient stage as they start with undercooling zero. This also further supports the argument that we had at the end of section 5.1, where we attributed the minor difference between macroscopic and mesoscopic values of $V_{t}$ to the presence of an initial transient stage.

\section{Conclusions}

A previously developed mesoscopic envelope model was used to perform three-dimensional simulations of equiaxed dendritic growth on a spatial scale that corresponds to a REV, which is used in developing volume-averaged macroscopic models. The first set of mesoscopic simulations were performed for isothermal growth at a large range of initial undercoolings and grain densities (including a single grain). The results were upscaled by averaging them over the REV. The upscaled results were examined in detail. It was found that the entire growth period can be divided into variable-sphericity and constant-sphericity stages based on the sign of the time derivative of the scaled primary arm length. During the variable-sphericity stage, the envelope growth is mainly due to the growth of the primary arms, while during the constant-sphericity stage it is mainly due to the growth of the secondary arms. It was also found that using the average undercooling in the extra-dendritic liquid in the Ivantsov solution significantly underpredicts the tip velocities.

For the first time in the field of solidification, the upscaled mesoscopic results were used to develop constitutive relations for macroscopic models of equiaxed solidification. Relations were proposed for the envelope sphericity, average growth velocity, far-field undercooling that needs to be used 
in the Ivantsov solution to accurately predict the primary tip velocities, and for the average diffusion length around the envelopes.

The constitutive relations were verified by comparing the predictions of the macroscopic model with the upscaled mesoscopic results for the isothermal and also for the new mesoscopic cases. These new cases involve external cooling and a recalescence in the cooling curves. For all the cases, the predicted macroscopic quantities were found to be in good agreement with the corresponding upscaled mesoscopic results. The minor difference between the two was attributed to a minor difference in the macroscopic and mesoscopic values of the primary tip velocity, and that was attributed to the presence of an initial transient stage in the mesoscopic simulations.

A necessary future work is comparing the predictions of the macroscopic model with the upscaled mesoscopic results for multiple grains in a random arrangement. Determining the final grain radius $R_{f}$ for this arrangement will be a key factor in macroscopic simulations. This can be achieved using methods such as grain count or linear intercept that are commonly used in microstructure analysis [34]. Extension of the mesoscopic model for multicomponent alloys and incorporating melt flow in the model are also of great interest.

\section{Acknowledgments}

This work was financially supported by NASA (NNX14AD69G) and by the French State through the program "Investment in the future" operated by the National Research Agency (ANR) and referenced by ANR-11 LABX-0008-01 (LabEx DAMAS). 


\section{References}

[1] I. Steinbach, C. Beckermann, B. Kauerauf, Q. Li, and J. Guo, "Three-dimensional modeling of equiaxed dendritic growth on a mesoscopic scale," Acta Materialia, vol. 47, no. 3, pp. 971-982, 1999.

[2] I. Steinbach, H.-J. Diepers, and C. Beckermann, "Transient growth and interaction of equiaxed dendrites," Journal of Crystal Growth, vol. 275, no. 3-4, pp. 624-638, 2005.

[3] C. Beckermann, and R. Viskanta, "Double-diffusive convection during dendritic solidification of a binary mixture," PhysicoChemical Hydrodynamics, vol. 10, no. 2, pp. 195-213, 1988.

[4] J. Ni, and C. Beckermann, "A volume-averaged two-phase model for transport phenomena during solidification," Metallurgical Transactions B, vol. 22B, pp. 349-361, 1991.

[5] C. Wang, and C. Beckermann, "A multiphase solute diffusion model for dendritic alloy solidification," Metallurgical and Materials Transactions A, vol. 24, no. 12, pp. 2787$2802,1993$.

[6] C. Beckermann, and C. Wang, "Multiphase/-scale modeling of alloy solidification," Annual Review of Heat Transfer, vol. 6, no. 6, 1995.

[7] C. Y. Wang, and C. Beckermann, "Equiaxed dendritic solidification with convection: Part I. Multiscale/multiphase modeling," Metallurgical and Materials Transactions A, vol. 27, no. 9, pp. 2754-2764, 1996.

[8] C. Wang, and C. Beckermann, "Equiaxed dendritic solidification with convection: Part II. Numerical simulations for an Al-4 Wt pet Cu alloy," Metallurgical and Materials Transactions A, vol. 27, no. 9, pp. 2765-2783, 1996.

[9] C. Beckermann, and C. Wang, "Equiaxed dendritic solidification with convection: Part III. Comparisons with NH4Cl-H2O experiments," Metallurgical and Materials Transactions A, vol. 27, no. 9, pp. 2784-2795, 1996. 
[10] M. Založnik, and H. Combeau, "Thermosolutal flow in steel ingots and the formation of mesosegregates," International Journal of Thermal Sciences, vol. 49, no. 9, pp. 1500-1509, 2010 .

[11] M. C. Schneider, and C. Beckermann, "Simulation of micro-/macrosegregation during the solidification of a low-alloy steel," ISIJ international, vol. 35, no. 6, pp. 665-672, 1995.

[12] H. Combeau, M. Založnik, and M. Bedel, "Predictive capabilities of multiphysics and multiscale models in modeling solidification of steel ingots and DC casting of aluminum," Jom, vol. 68, no. 8, pp. 2198-2206, 2016.

[13] C. Beckermann, "Modelling of macrosegregation: applications and future needs," International Materials Reviews, vol. 47, no. 5, pp. 243-261, 2002.

[14] P. Delaleau, C. Beckermann, R. H. Mathiesen, and L. Arnberg, "Mesoscopic simulation of dendritic growth observed in X-ray video microscopy during directional solidification of Al-Cu alloys," ISIJ international, vol. 50, no. 12, pp. 1886-1894, 2010.

[15] Y. Souhar, V. F. De Felice, C. Beckermann, H. Combeau, and M. Založnik, “Threedimensional mesoscopic modeling of equiaxed dendritic solidification of a binary alloy," Computational Materials Science, vol. 112, pp. 304-317, 2016.

[16] A. Melendez, and C. Beckermann, "Measurements of dendrite tip growth and sidebranching in succinonitrile-acetone alloys," Journal of Crystal Growth, vol. 340, no. 1, pp. 175-189, 2012.

[17] Ø. Nielsen, A. Mo, B. Appolaire, and H. Combeau, "Measurements and modeling of the microstructural morphology during equiaxed solidification of Al-Cu alloys," Metallurgical and Materials Transactions A, vol. 32, no. 8, pp. 2049-2060, 2001.

[18] M. A. Martorano, C. Beckermann, and C.-A. Gandin, "A solutal interaction mechanism for the columnar-to-equiaxed transition in alloy solidification," Metallurgical and Materials Transactions A, vol. 34, no. 8, pp. 1657-1674, 2003. 
[19] B. Appolaire, H. Combeau, and G. Lesoult, "Modeling of equiaxed growth in multicomponent alloys accounting for convection and for the globular/dendritic morphological transition," Materials Science and Engineering: A, vol. 487, no. 1-2, pp. $33-45,2008$.

[20] M. Wu, and A. Ludwig, "Modeling equiaxed solidification with melt convection and grain sedimentation-I: Model description," Acta Materialia, vol. 57, no. 19, pp. 5621-5631, 2009.

[21] M. Wu, and A. Ludwig, "Modeling equiaxed solidification with melt convection and grain sedimentation-II. Model verification,” Acta Materialia, vol. 57, no. 19, pp. 5632-5644, 2009.

[22] M. Rappaz, and P. Thevoz, "Solute diffusion model for equiaxed dendritic growth," Acta Metallurgica, vol. 35, no. 7, pp. 1487-1497, 1987.

[23] M. Wu, A. Fjeld, and A. Ludwig, "Modelling mixed columnar-equiaxed solidification with melt convection and grain sedimentation-Part I: Model description," Computational Materials Science, vol. 50, no. 1, pp. 32-42, 2010.

[24] M. Wu, A. Ludwig, and A. Fjeld, "Modelling mixed columnar-equiaxed solidification with melt convection and grain sedimentation-Part II: Illustrative modelling results and parameter studies," Computational Materials Science, vol. 50, no. 1, pp. 43-58, 2010.

[25] C. Wang, and C. Beckermann, "Prediction of columnar to equiaxed transition during diffusion-controlled dendritic alloy solidification," Metallurgical and Materials Transactions A, vol. 25, no. 5, pp. 1081-1093, 1994.

[26] R. Trivedi, and W. Kurz, "Dendritic growth," International Materials Reviews, vol. 39, no. 2, pp. 49-74, 1994.

[27] J. A. Dantzig, and M. Rappaz, Solidification: EPFL press, 2009.

[28] M. Glicksman, M. Koss, and E. Winsa, "Dendritic growth velocities in microgravity," Physical review letters, vol. 73, no. 4, pp. 573, 1994. 
[29] V. Pines, A. Chait, M. Zlatkowski, and C. Beckermann, "Equiaxed dendritic solidification in supercooled melts," Journal of Crystal Growth, vol. 197, no. 1-2, pp. 355-363, 1999.

[30] B. J. Spencer, and H. E. Huppert, "Steady-state solutions for an array of stronglyinteracting needle crystals in the limit of small undercooling," Journal of Crystal Growth, vol. 148, no. 3, pp. 305-323, 1995.

[31] G. Zimmermann, L. Sturz, H. Nguyen-Thi, N. Mangelinck-Noël, Y. Li, C.-A. Gandin, R. Fleurisson, G. Guillemot, S. Mcfadden, and R. P. Mooney, "Columnar and Equiaxed Solidification of Al-7 wt.\% Si Alloys in Reduced Gravity in the Framework of the CETSOL Project," JOM, vol. 69, no. 8, pp. 1269-1279, 2017.

[32] Y. Souhar, V. F. De Felice, M. Založnik, H. Combeau, and C. Beckermann, "The role of the stagnant-film thickness in mesoscopic modeling of equiaxed grain envelopes," IOP Conf. Series: Materials Science and Engineering. p. 012014.

[33] M. Torabi Rad, and C. Beckermann, "Validation of A Model for the Columnar to Equiaxed Transition with Melt Convection," CFD Modeling and Simulation in Materials Processing 2016, pp. 85-92: Springer, 2016.

[34] E. E. Underwood, "Quantitative stereology for microstructural analysis," Microstructural Analysis, pp. 35-66: Springer, 1973. 


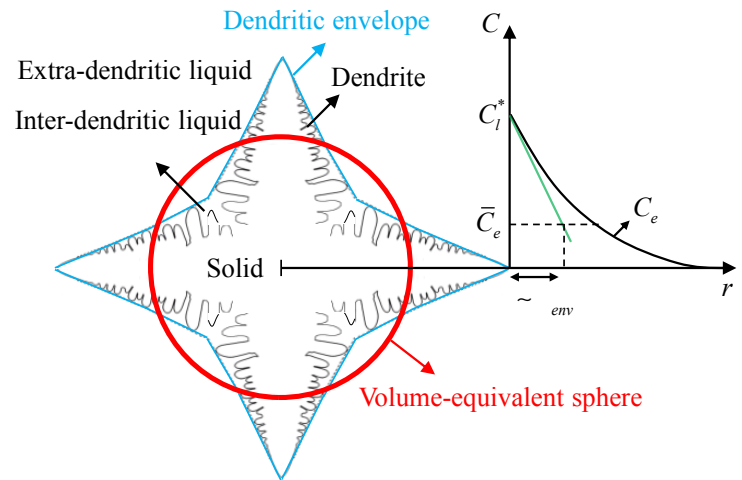

(a)

Figure 1. Two-dimensional schematic of a single equiaxed dendrite growing into an essentially infinite medium; the dendritic envelope and volume-equivalent sphere; regions of solid, interdendritic liquid, and extra-dendritic liquid; and an schematic of the solute distribution in the extra-dendritic liquid ahead of the primary tip along with the tangent to the profile at the position of the tip; $r$ represents the distance from the dendrite center. 


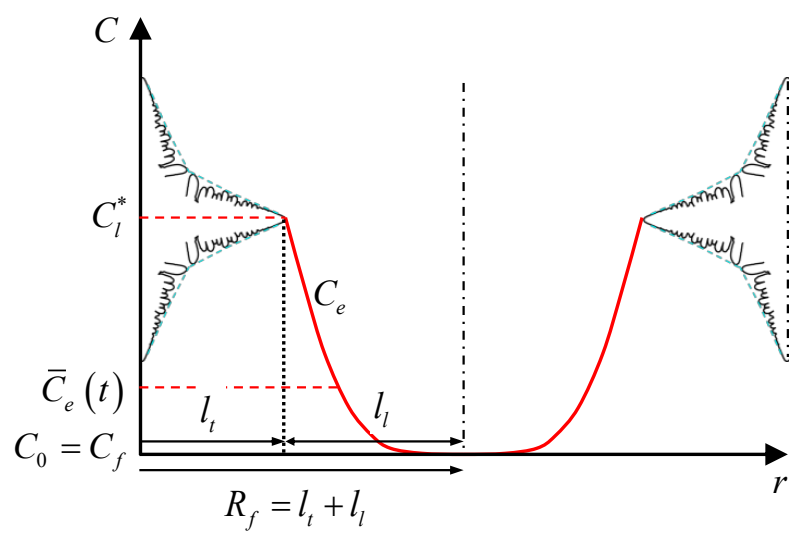

(a)

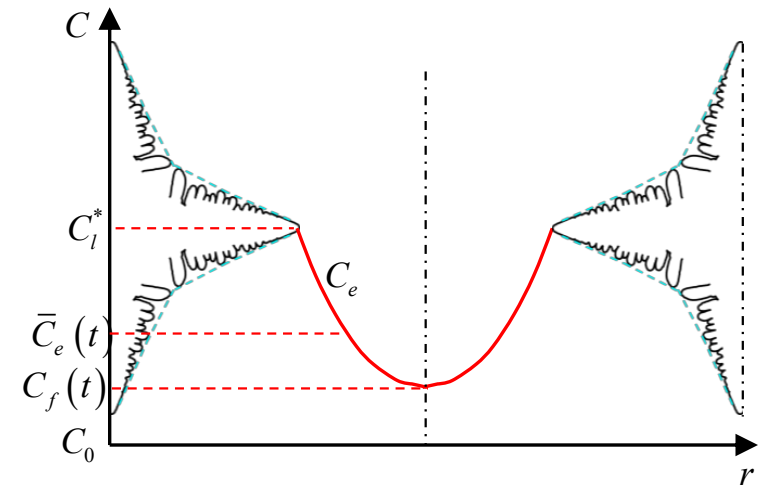

(b)

Figure 2. Schematics of the extra-dendritic liquid solute concentration profiles ahead of the primary tips of two adjacent dendrites, at a time instance in the (a) non-interacting stage and (b) interacting stage; $r$ represents the distance from the center of the left dendrite. 


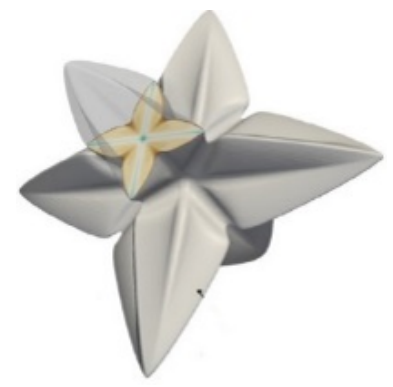

(a)

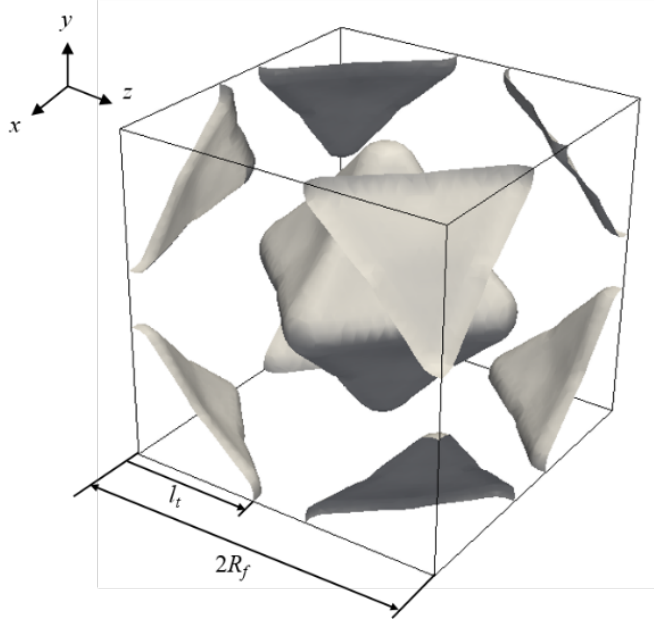

(b)

Figure 3. Mesoscopic grain envelopes for (a) a single grain and (b) multiple grains in the $\mathrm{BCC}$ arrangement with the primary arms growing along the $x, y$, and $z$ axes. 


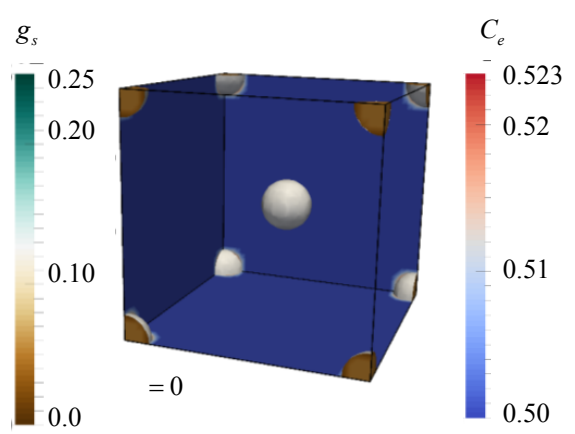

(a)

gs

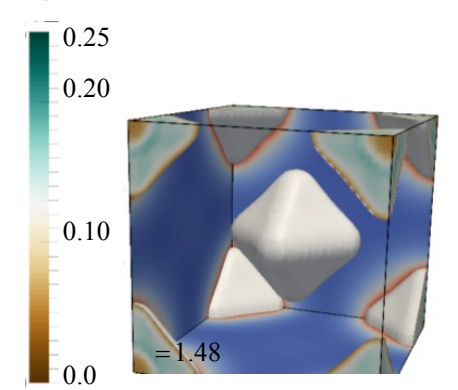

(c)

$g_{s}$

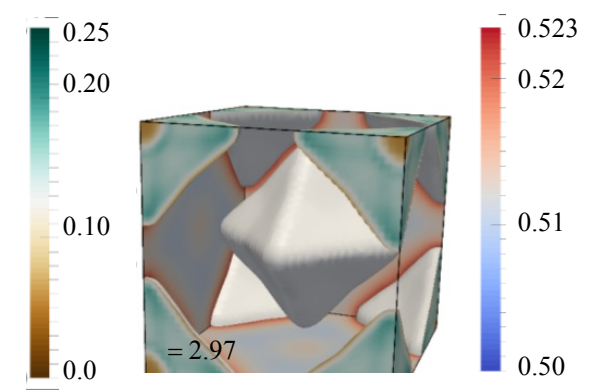

(e)

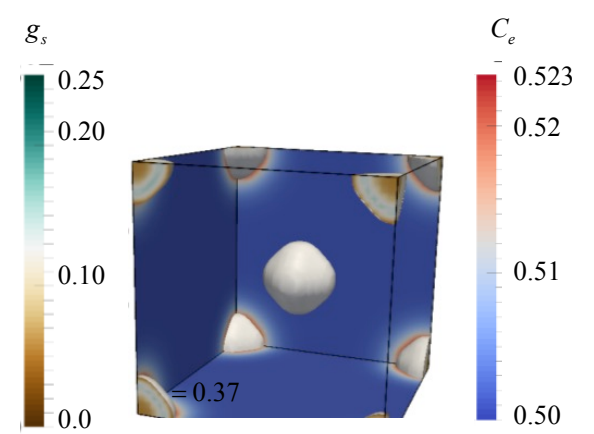

(b)

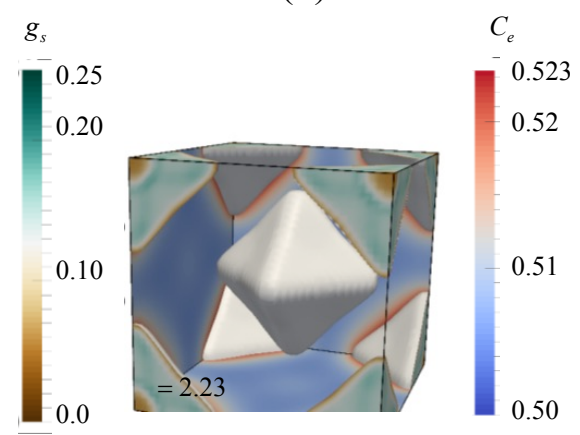

(d)

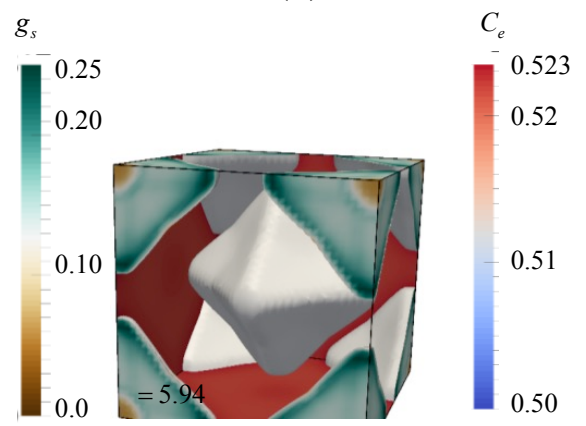

(f)

Figure 4. Mesoscopic simulation results showing solid fraction $g_{s}$ (plotted in the interior of the envelopes) and solute concentration in the extra-dendritic liquid $C_{e}$ (plotted in the exterior of the envelopes) at different non-dimensional times $=t V_{I v}^{2}\left(\begin{array}{l}0 \\ 0\end{array}\right) / D_{l}:$ (a) $=0$, (b) 0.37 , (c) 1.48 , (d) 2.23 , (e) 2.97, and (f) 5.94. This simulations is for the isothermal case with low undercooling ( $\left.{ }_{0}=0.05\right)$ and high grain density $\left(R_{f} / D_{l} / V_{l v}\left(\begin{array}{l}0 \\ 0\end{array}\right)=4.03\right)$. 


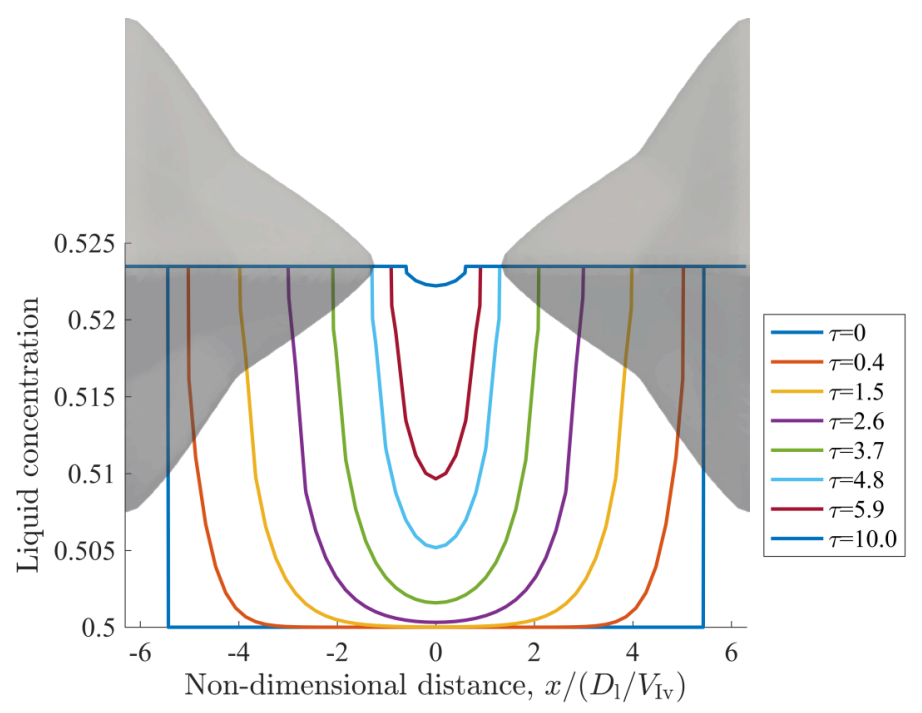

Figure 5. Mesoscopic simulation results showing profiles of liquid solute concentration along the line connecting the primary arms of two dendrites growing towards each other. Different curves show the profiles at different times. 


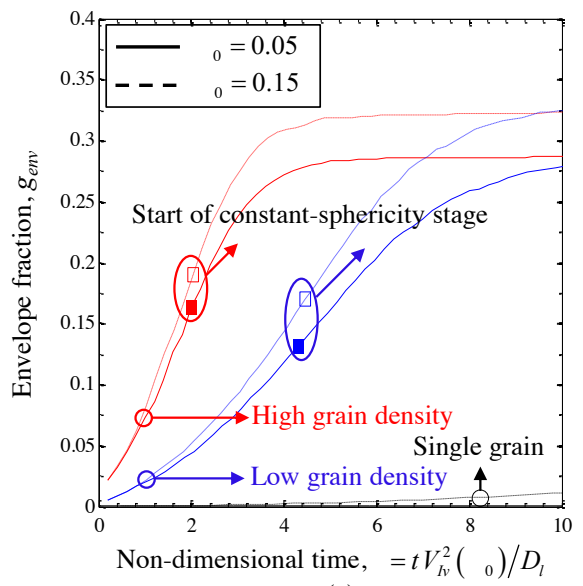

(a)

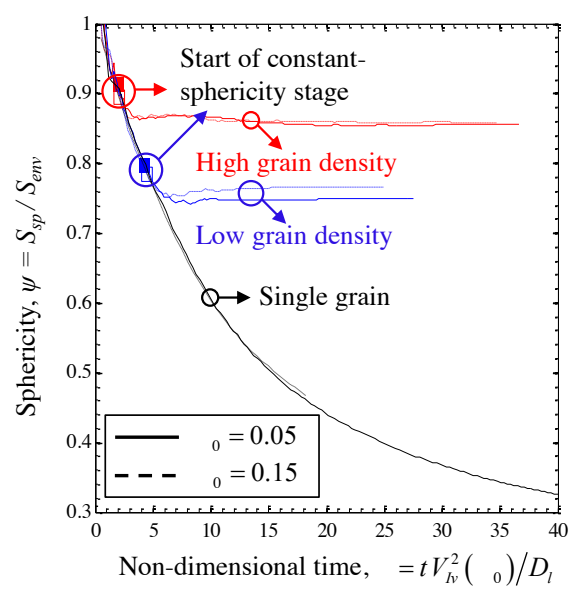

(c)

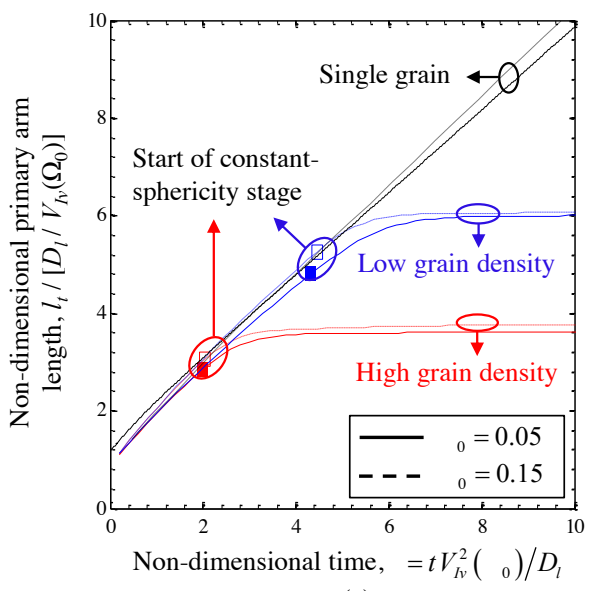

(e)

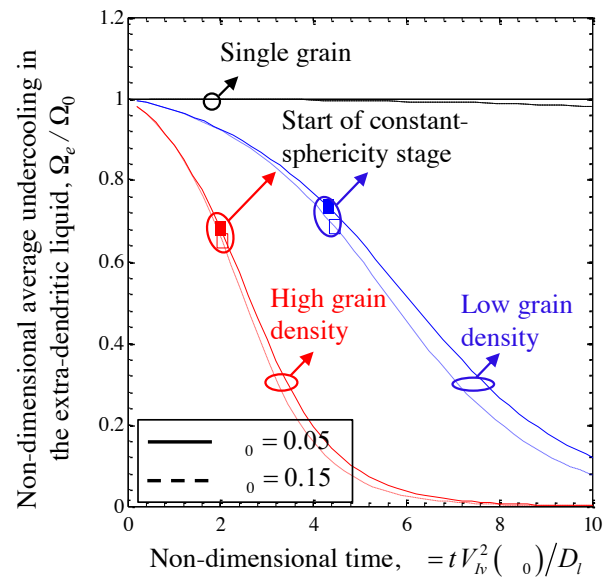

(b)

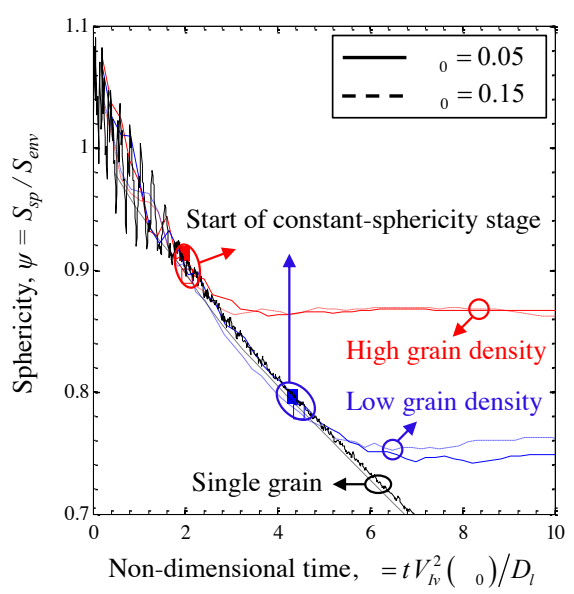

(d)

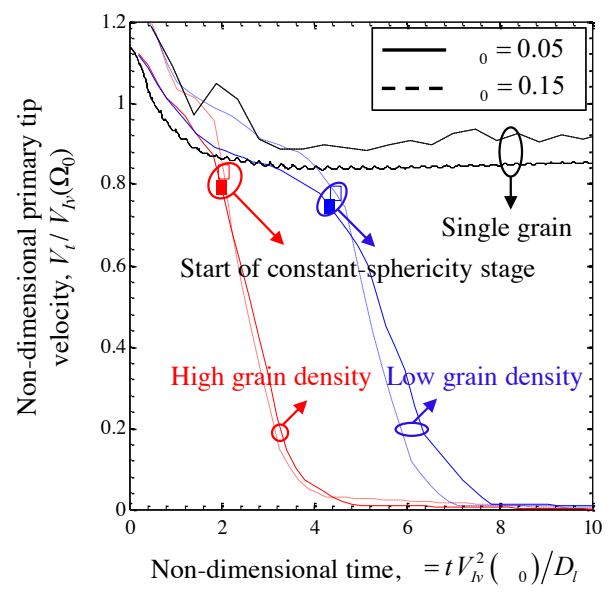

(f) 


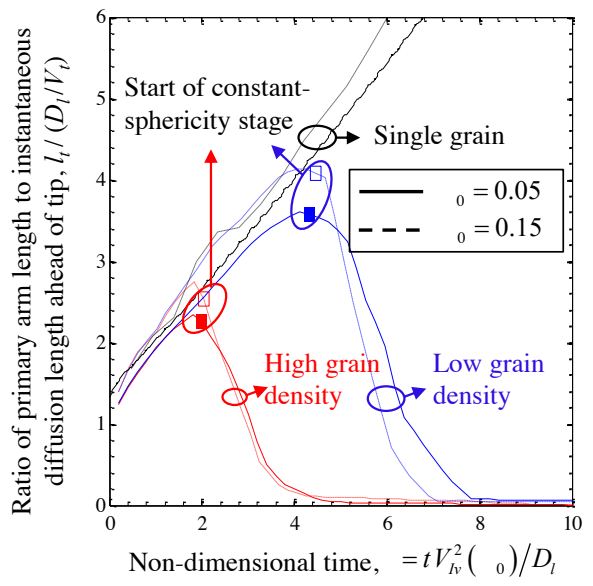

(g)

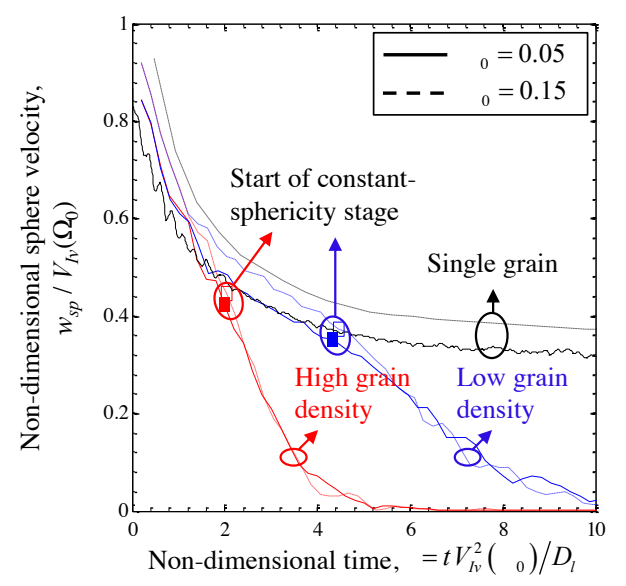

(i)

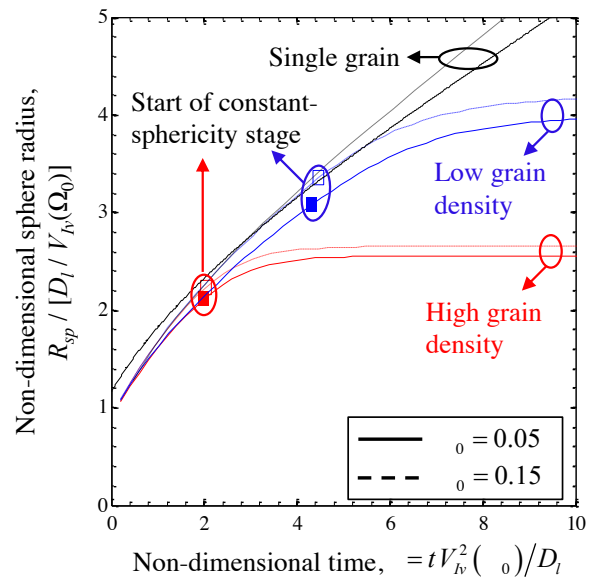

(h)

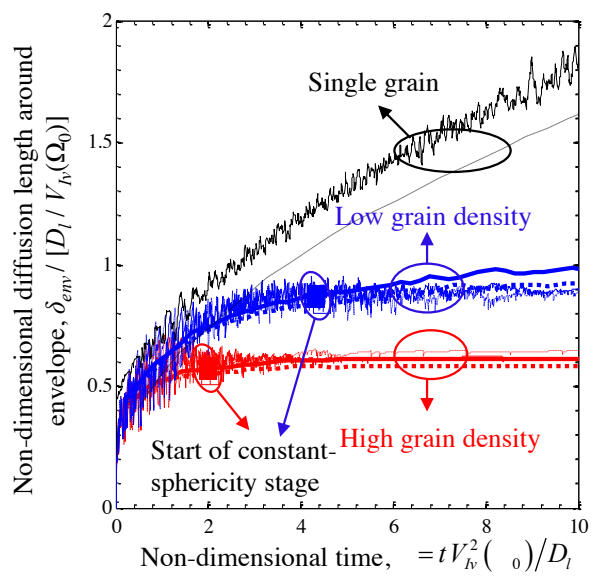

(j)

Figure 6. (a-j) Upscaled mesoscopic results plotted as a function of non-dimensional time. High and low grain density cases correspond to $R_{f} / D_{l} / V_{I v}\left({ }_{0}\right)=4.03$ and 6.31 , respectively. 


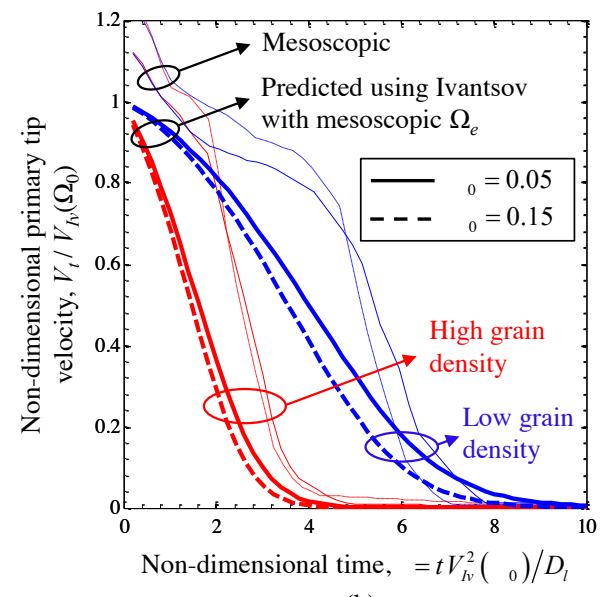

(k)

Figure 7. Comparison between the mesoscopic primary tip velocities and the Ivantsov primary tip velocities corresponding to the average undercooling in the extra-dendritic liquid. 


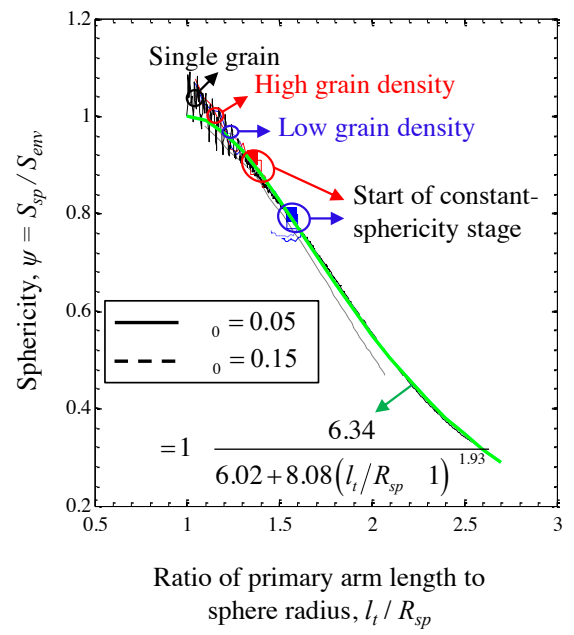

Figure 8 . The envelope sphericity as a function of the ratio of the primary arm length to sphere radius. The green curve represents our curve fit. 


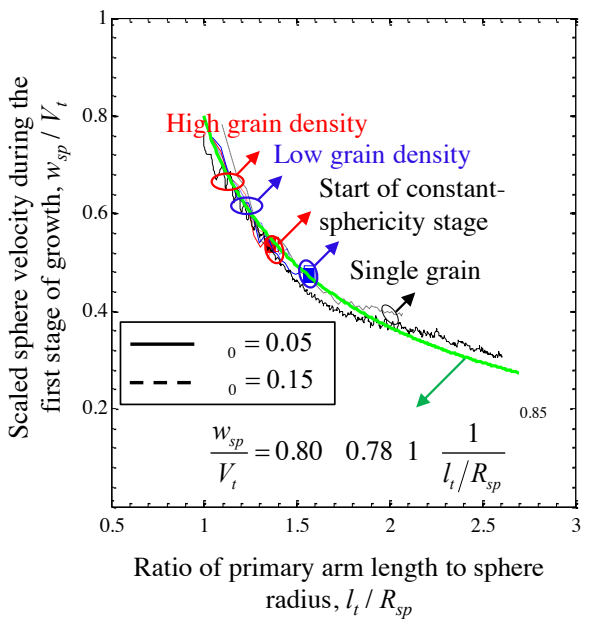

(a)

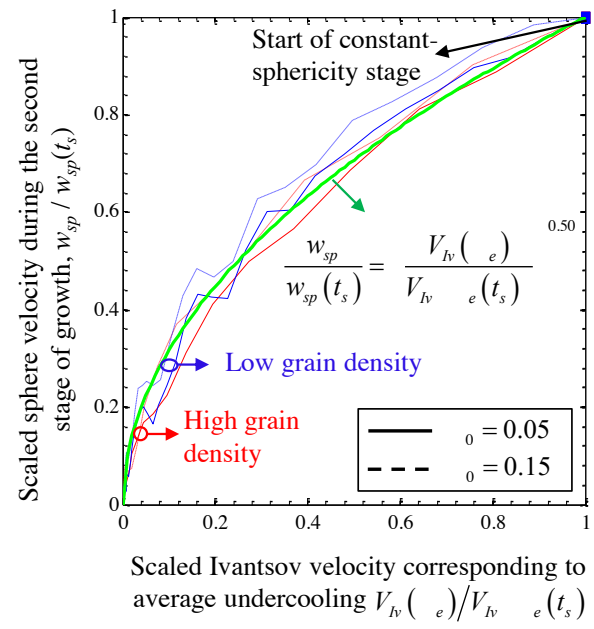

(b)

Figure 9. Scaled sphere growth velocity during (a) the variable-sphericty stage of growth as a function of the ratio of primary arm length to sphere radius, and during (b) the constantsphericity stage of growth as a function of scaled Ivantsov velocity corresponding to the average undercooling in the extra-dendritic liquid. The green curves represent our curve fits. 


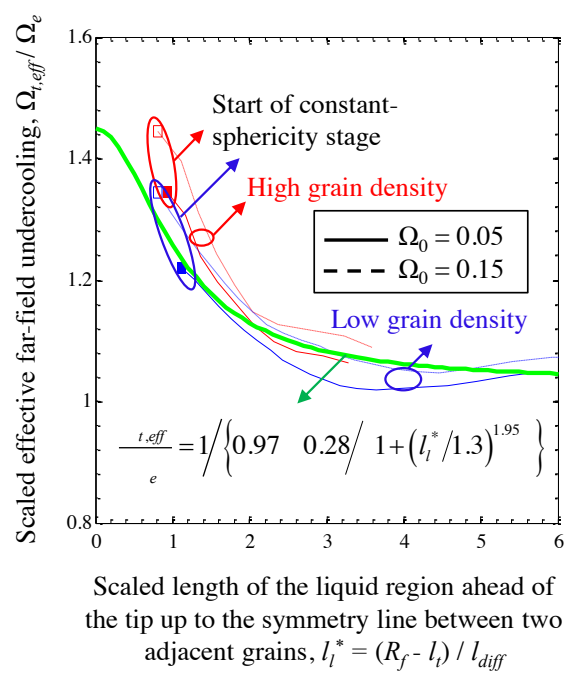

Figure 10. The scaled effective far-field undercooling as a function of the scaled length of the liquid region ahead of the tip up to the symmetry line between two adjacent grains. The green curve represents our curve fit. 


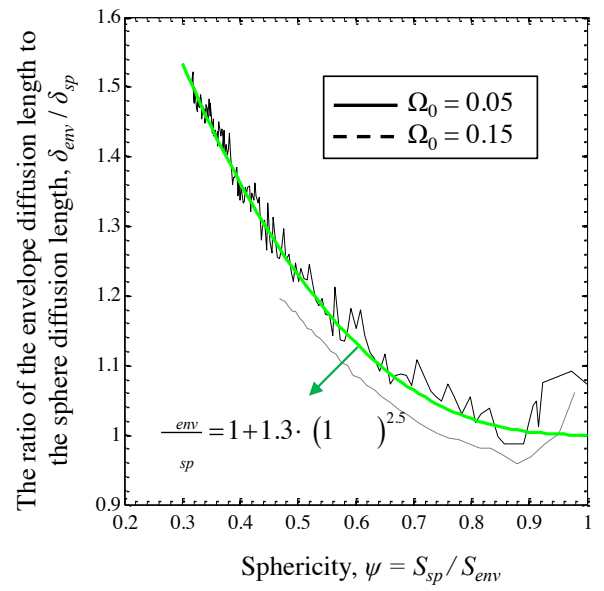

Figure 11. The ratio of the envelope diffusion length to the sphere diffusion length as a function of the envelope sphericity for a single grain at two different initial undercoolings. The green curve represents our curve fit. 


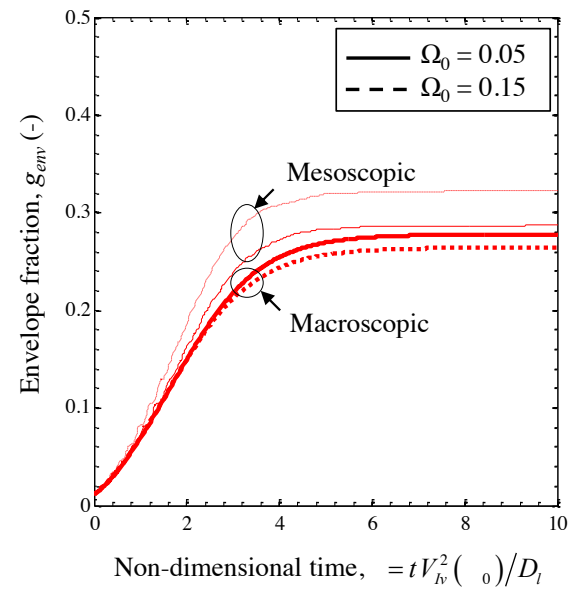

(a)

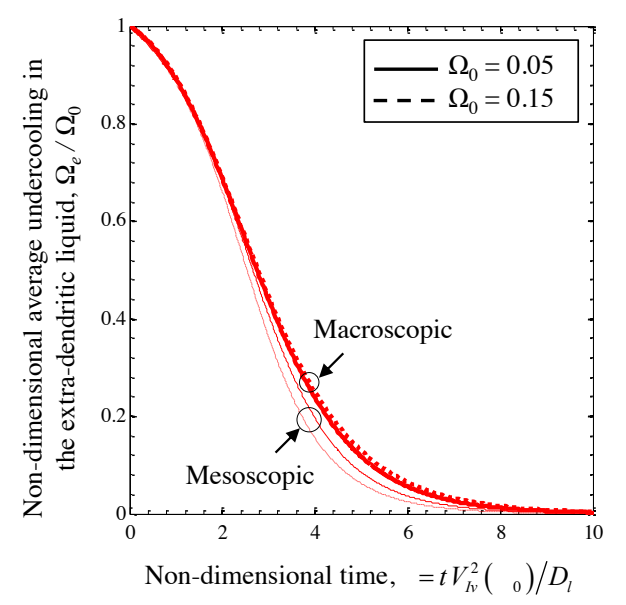

(c)

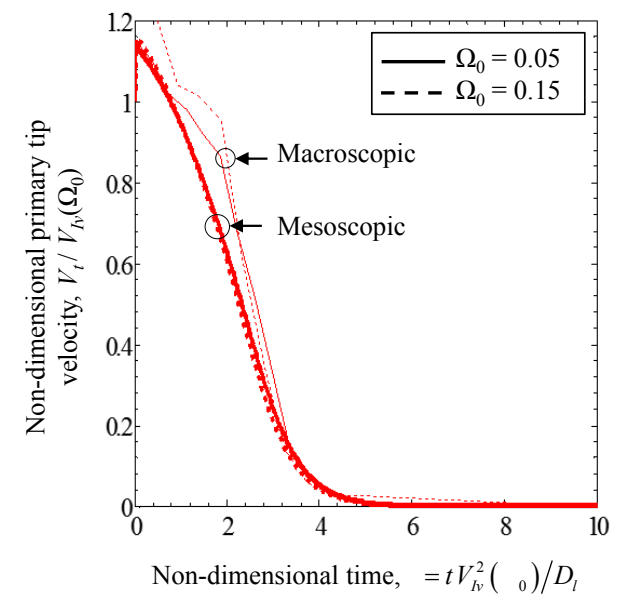

(e)

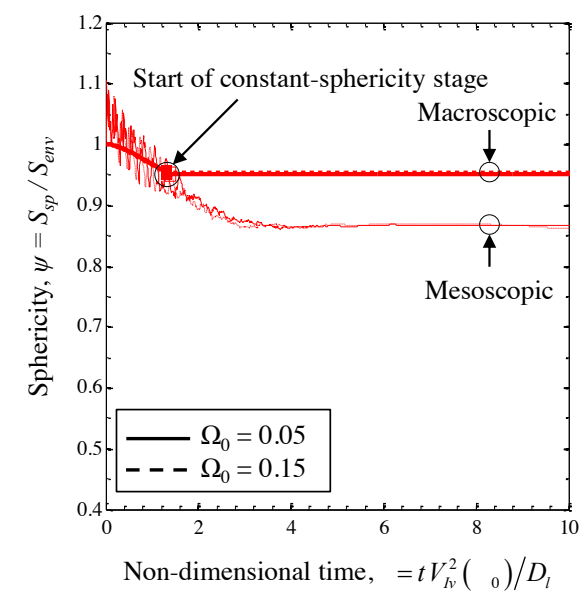

(b)

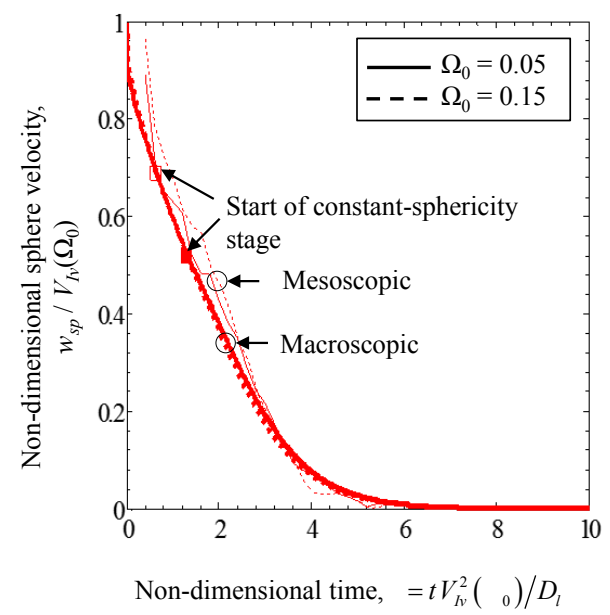

(d)

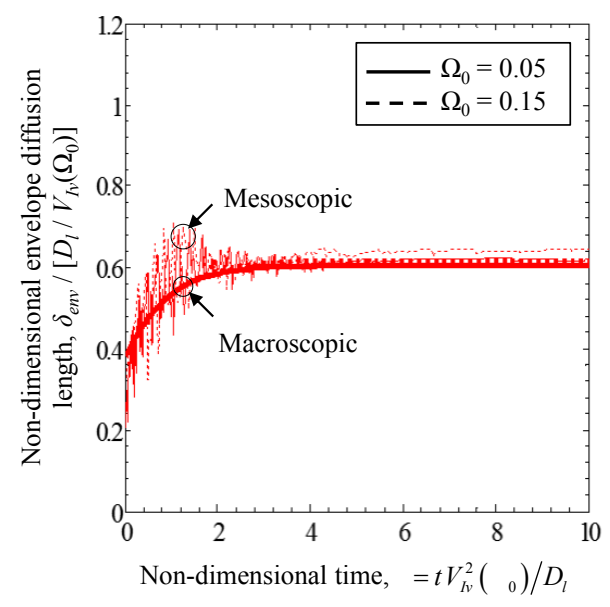

(f)

Figure 12. Comparison between the mesoscopic and macroscopic quantities plotted as a funciton of non-dimensional time. This comparison is for the isothermal case with high grain density and low undercooling: $R_{f} / D_{l} / V_{I v}\left({ }_{0}\right)=4.03$. 


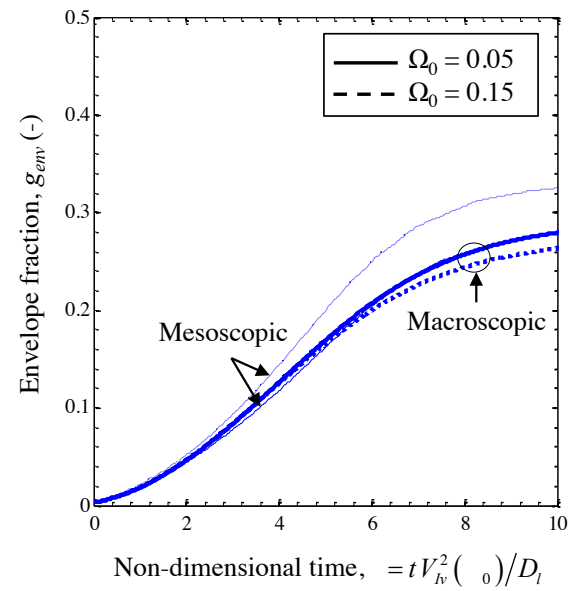

(a)

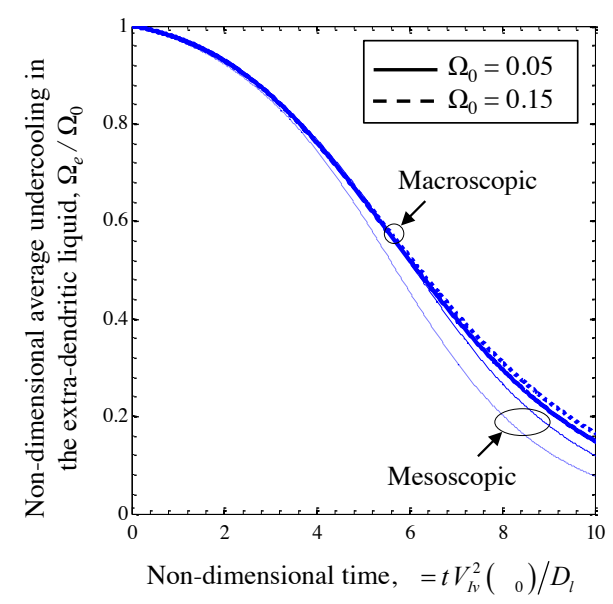

(c)

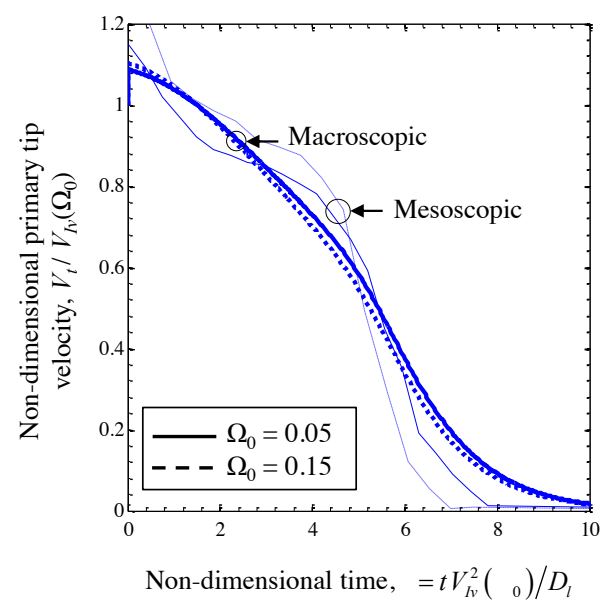

(e)

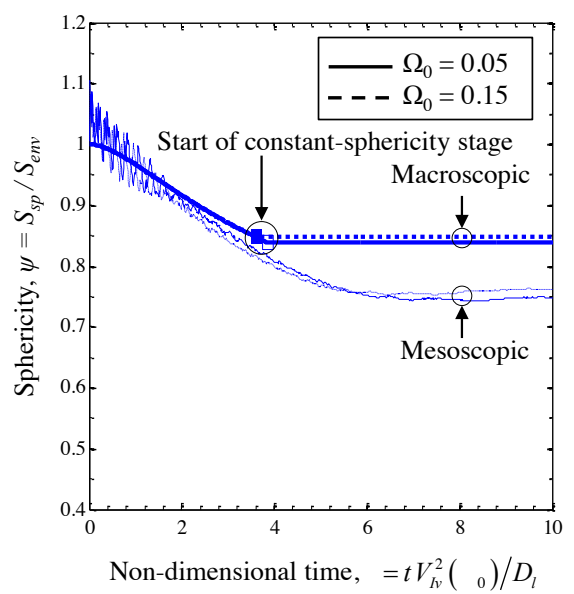

(b)

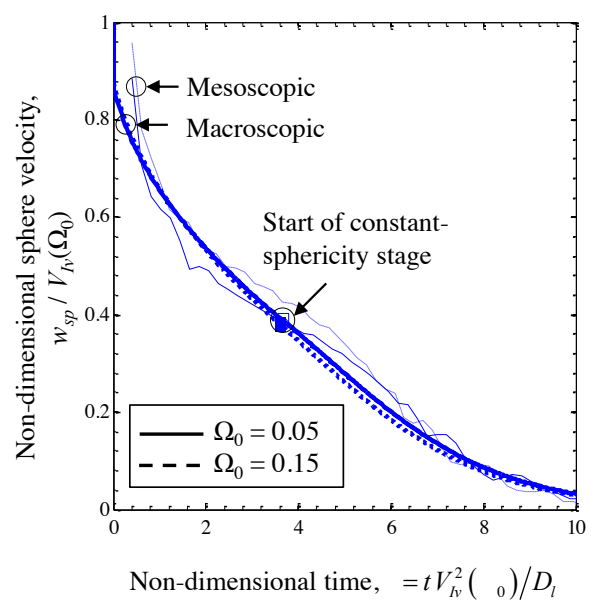

(d)

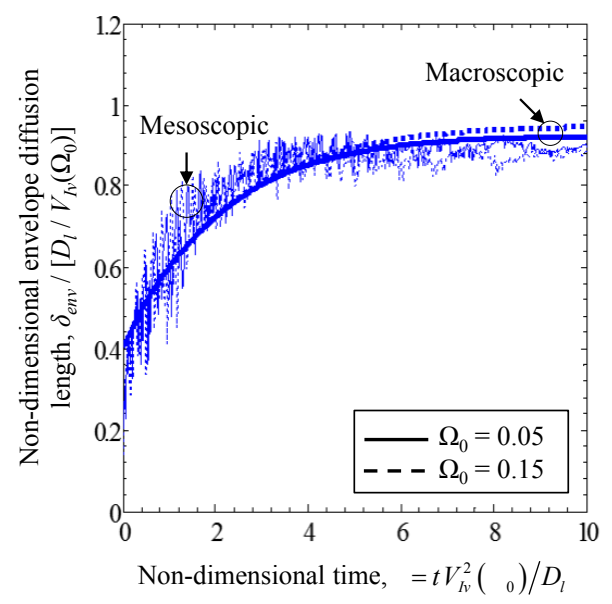

(f)

Figure 13. Comparison between the mesoscopic and macroscopic quantities plotted as a function of non-dimensional time. This comparison is for the isothermal case with low grain density and low undercooling: $R_{f} / D_{l} / V_{I v}\left({ }_{0}\right)=6.31$. 


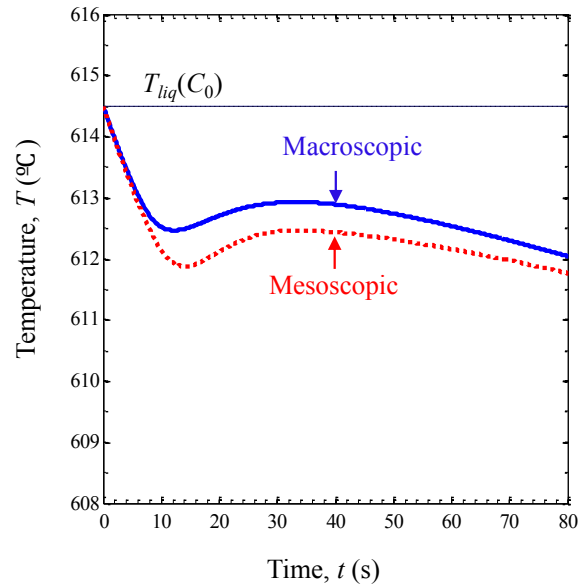

(a)

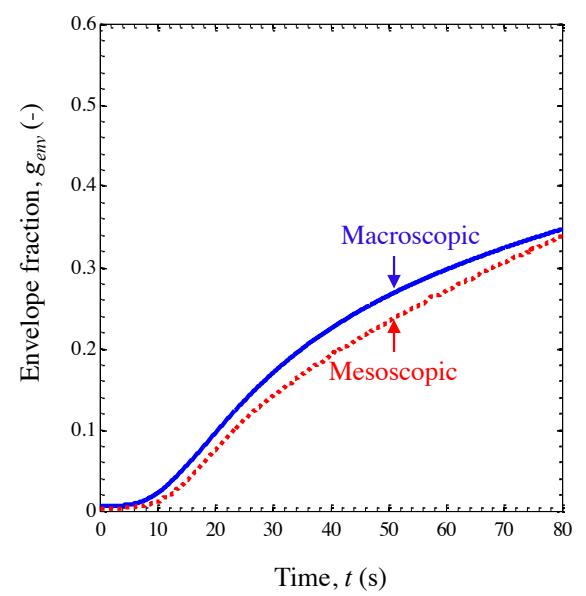

(c)

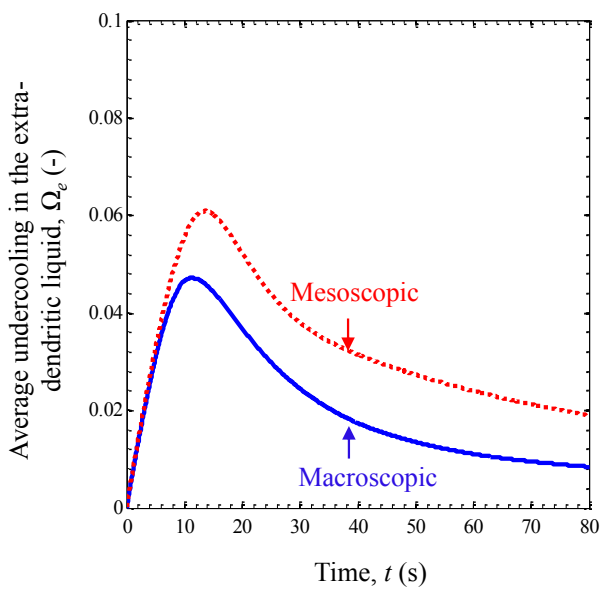

(e)

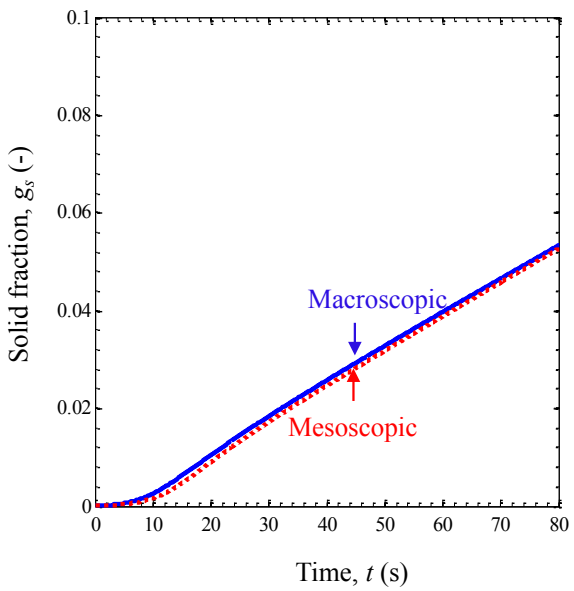

(b)

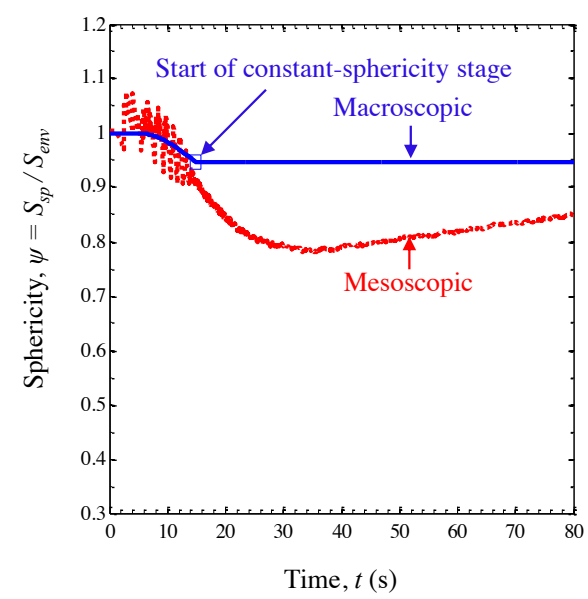

(d)

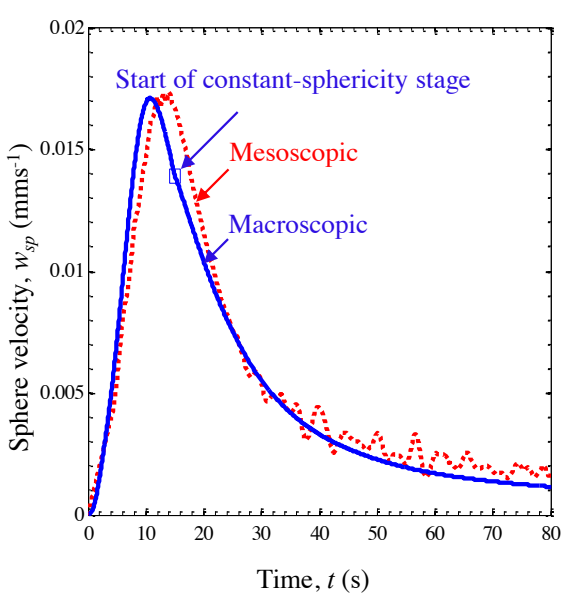

(f) 


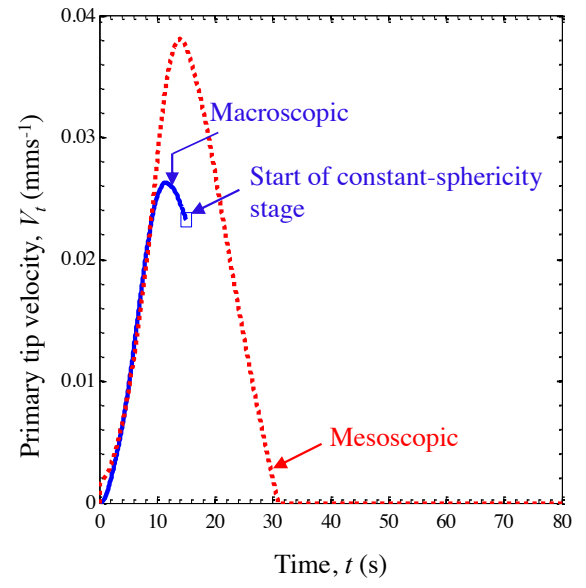

(g)

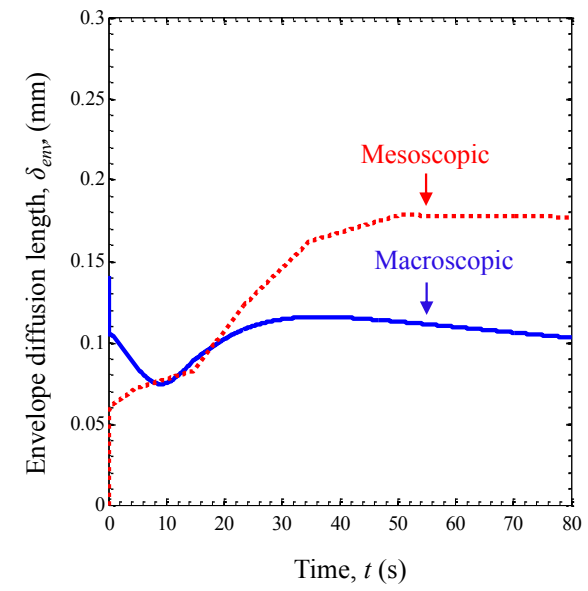

(h)

Figure 14. Comparison between the mesoscopic and macroscopic quantities plotted as a function of time. This comparison is for the recalescence case with high grain density: $R_{f}=0.8 \mathrm{~mm}$. 


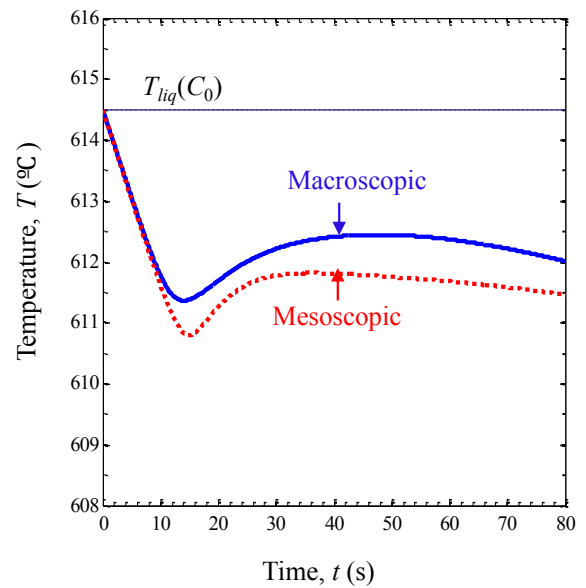

(a)

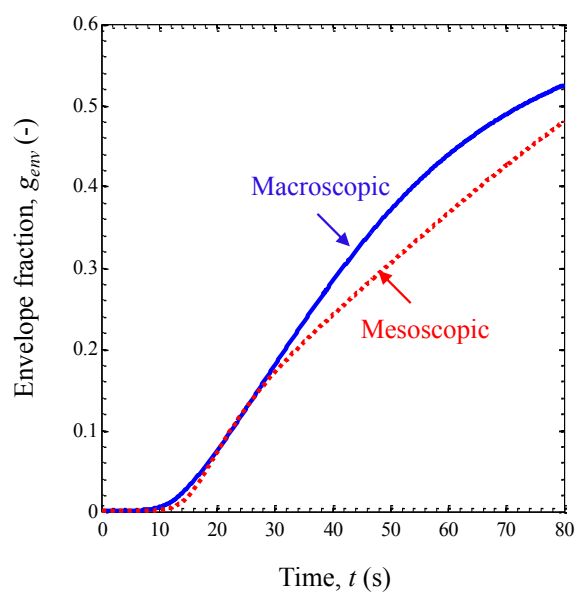

(c)

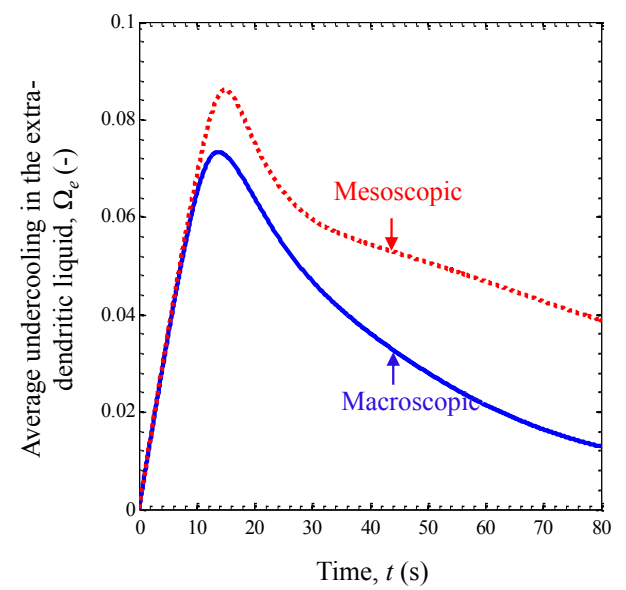

(e)

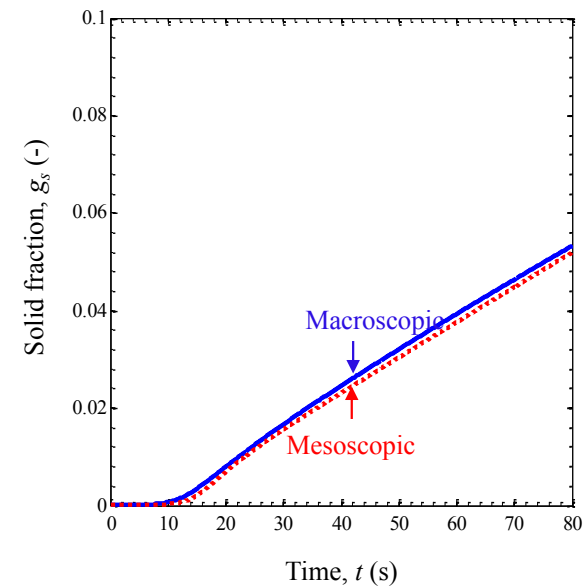

(b)

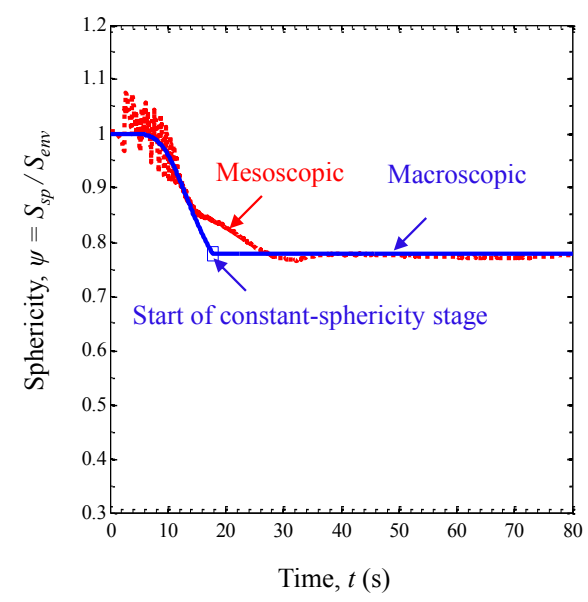

(d)

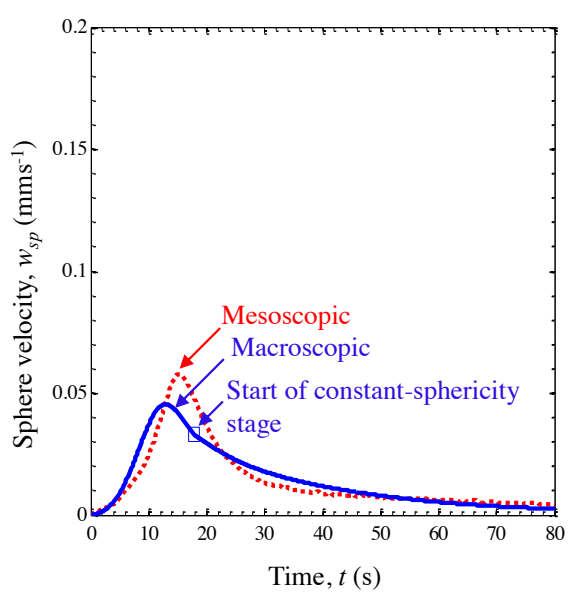

(f) 


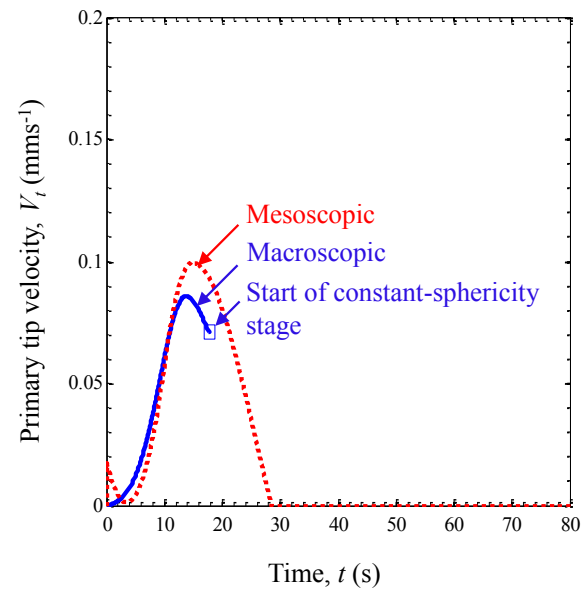

(g)

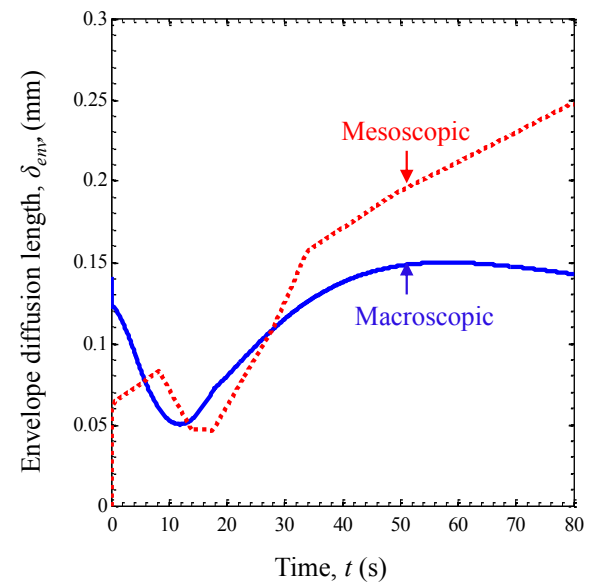

(h)

Figure 15. Comparison between the mesoscopic and macroscopic quantities plotted as a function of time. This comparison is for the recalescence case with intermediate grain density: $R_{f}=1.6 \mathrm{~mm}$. 


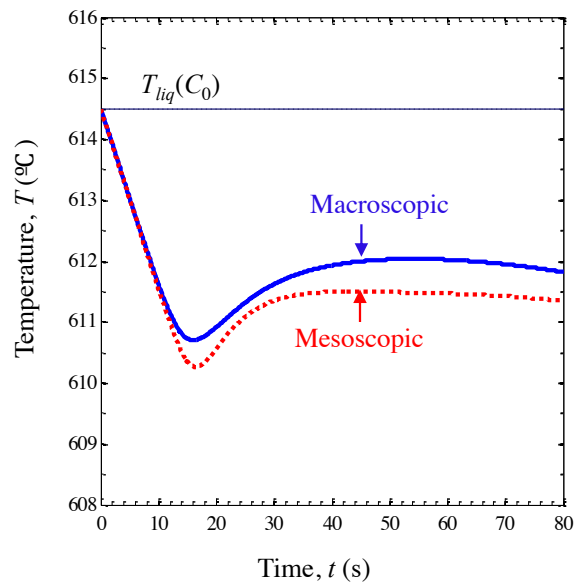

(a)

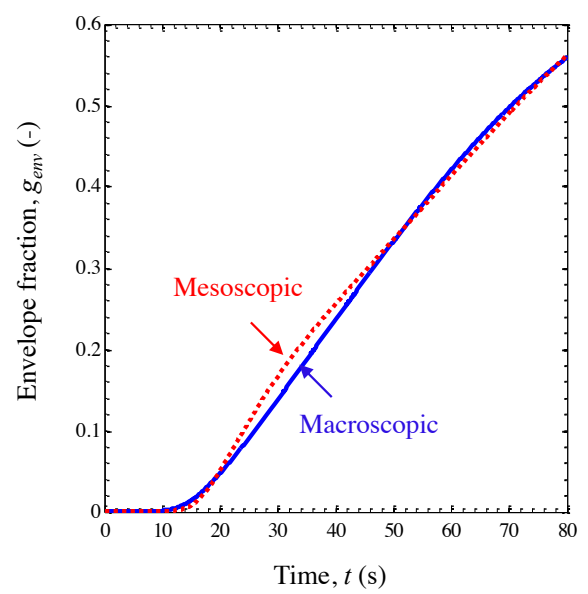

(c)

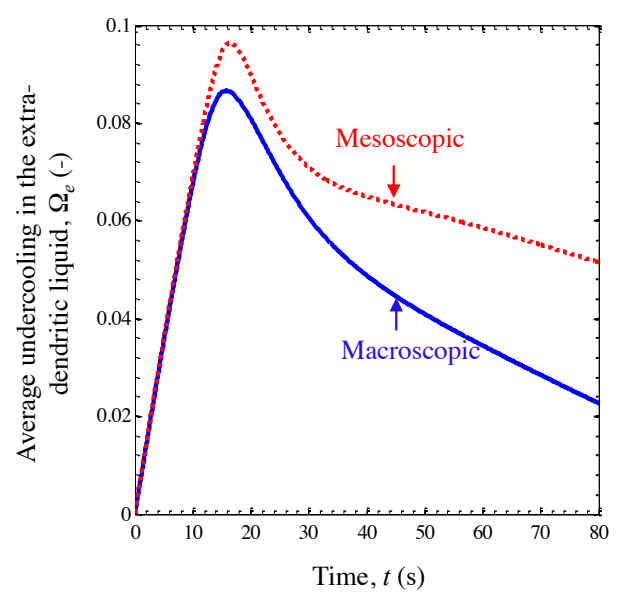

(e)

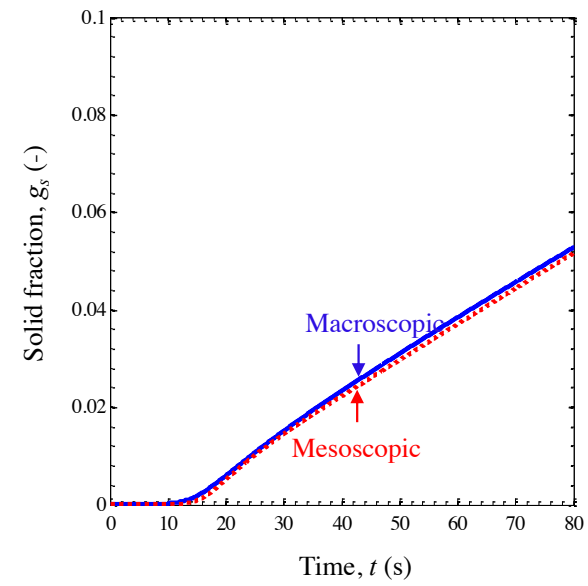

(b)

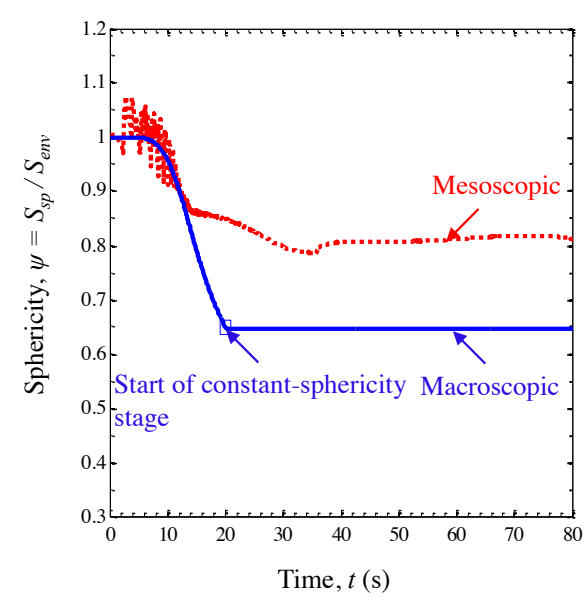

(d)

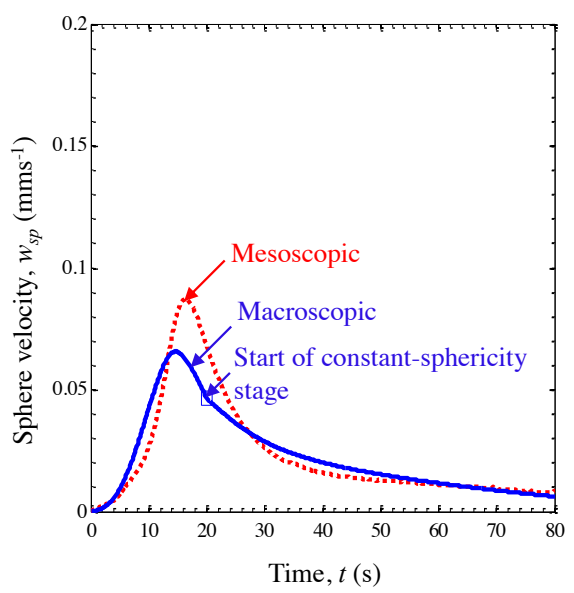

(f) 


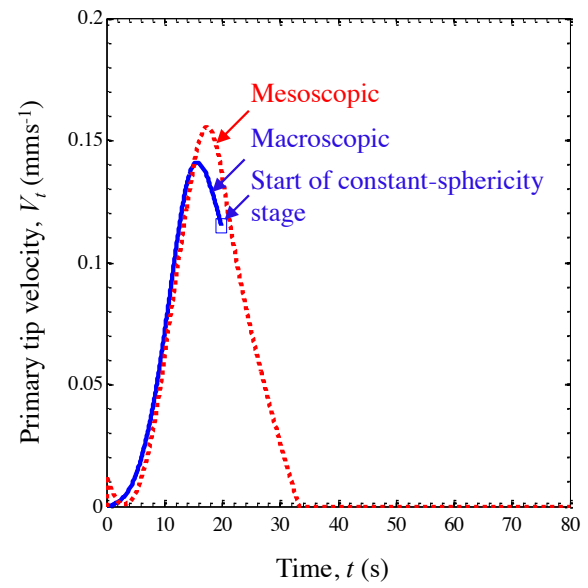

(g)

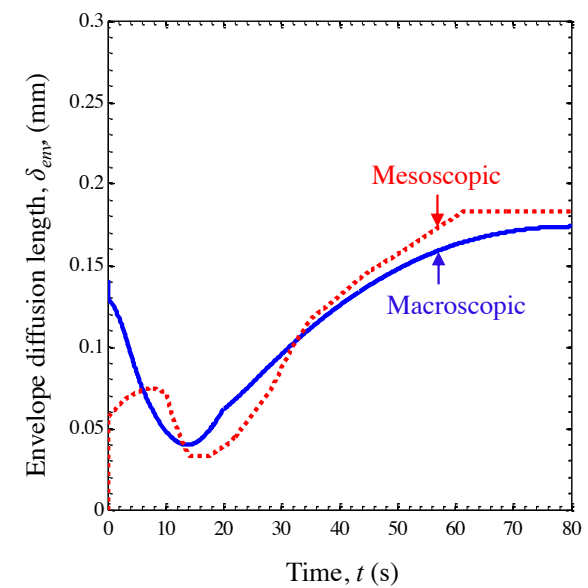

(h)

Figure 16. Comparison between the mesoscopic and macroscopic quantities plotted as a function of time. This comparison is for the recalescence case with low grain density: $R_{f}=2.4 \mathrm{~mm}$. 